\title{
El lugar de la interferencia en la percepción de estímulos simultáneos
}

\section{John Duncan}

\author{
MRC Applied Psychology Unit, Cambridge, England \\ Funnte: Psychological Review, 87, 272-300, 1980.
}

\section{TEORIAS DE LA ATENCION}

¿Qué es lo que limita la capacidad de las personas para identificar (dividir la atención entre) varios estímulos a la vez? Las explicaciones que se basan en la selección temprana (early selection) distinguen entre dos sistemas de análisis perceptual. En primer lugar, un sistema paralelo (preatencional) extrae las características simples del estímulo, como por ejemplo el color (visión) o la voz (audición). En segundo lugar, hay un sistema de capacidad limitada (atencional) que es responsable del análisis de la forma y del significado. Este segundo sistema es el único que tiene un límite en su capacidad para tratar con varios estímulos a la vez. La elección de los estímulos que pasan del primer sistema al segundo puede hacerse en base a cualquiera de las características que ya ha analizado el primer sistema. Por ejemplo, una persona podría querer identificar sólo las palabras que dice una cierta voz. La voz la analiza el primer sistema; sólo las palabras que emite la voz elegida necesitan pasar al segundo siste- ma (de capacidad limitada) para que su forma sea analizada. Broadbent (1971), Treisman (1964 a, 1964 b) y Neisser (1967) han ofrecido explicaciones de este tipo.

Las explicaciones que se basan en una selección tardia (late selection) distinguen también entre un primer sistema paralelo y otro sistema con capacidad limitada. La diferencia está en la suposición de que en el primer sistema se extraen tanto la forma y el significado como las características estimulares más simples, como el color o la voz. Se han hecho varias interpretaciones del segundo sistema, como por ejemplo, la consolidación en la memoria a corto plazo (Shiffrin, 1975), o la formación de un percepto coordinado a partir de características estimulares discretas (Allport, 1977). Lo importante es que incluso la forma y el significado se extraen en paralelo de todos los estímulos, de manera que éstos se pueden seleccionar en base a estas características para que pasen del primer sistema al segundo. A partir de Deutsch y Deutsch (1963) muchos autores han adoptado posiciones de este tipo (e.g., Allport, 1977; Hoffman, 1978; Keele, 
1973; Posner, 1978; Shiffrin y Schneider, 1977).

El objetivo de este artículo es desarrollar nuevos argumentos en defensa de la selección tardía, basados en parte en la literatura $y$ en parte en experimentos nuevos.

\section{ESTIMULOS OBJETIVOS EN LA ATENCION DIVIDIDA}

Comenzaré considerando la influencia que la división de la atención tiene en algunos tipos de experimentos de búsqueda de objetivos (targets). Un punto importante que surgirá es que no tiene especial relevancia el que la tarea requiera discriminación de formas. En algunas situaciones los decrementos en rendimiento que se producirán por la división de la atención serán pequeños, tanto si se requiere discriminación de formas como si no, mientras que en otras situaciones se producirán decrementos mayores. No hay razón para considerar que el procesamiento de la forma tenga propiedades especiales.

\section{Búsqueda de un único objetivo}

Un conjunto importante de experimentos se refiere a la búsqueda visual. Se busca un objetivo preespecificado entre un conjunto de caracteres (normalmente letras o dígitos). Por ahora me ceñiré a las situaciones en las que los objetivos y no-objetivos, o pertenecen a categorías bien aprendidas y distintas (e.g., búsqueda de objetivos dígitos entre letras no-objetivos) o se distinguen por alguna característica física sencilla, como por ejemplo el color. Es sabido que en estas circunstancias la relación entre los tiempos de reacción, o la exactitud, y el número de signos de la muestra sugiere que hay algún tipo de procesamiento paralelo (Egeth, Jonides y Wall, 1972; Jonides y Gleitman, 1972; Schneider y Shiffrin, 1977; Treisman, Sykes y Gelade, 1977). Por tanto no hay un límite evidente en la capacidad para dividir la atención entre estímulos.

Incluso en tareas muy simples y muy practicadas suele darse algún pequeño decremento en el rendimiento (disminución de la velocidad o la exactitud) a medida que se aumenta el tamaño de la muestra (Corcoran y Jackson, 1977; Egeth et al., 1972). Pero esto suele atribuirse a otras causas que no tienen nada que ver con la atención. En mu- chos experimentos los caracteres están agrupados en conjuntos mayores (aumentando la probabilidad de que se produzca un enmascaramiento periférico), o dispersos por la periferia visual. Hay también un importante argumento estadístico (Eriksen y Spencer, 1969). A medida que aumenta el tamaño de la exposición, aumenta la probabilidad de que un no-objetivo se confunda con un objetivo. Esto se da, no porque cada signo se examine con menos exactitud, sino sencillamente por razones de probabilidad. Si (para exposiciones de cualquier tamaño) la probabilidad de tomar un no-objetivo por un objetivo es $p$, la probabilidad de que no se den estos errores $(1-p)^{\mathrm{n}}$ siendo $\mathrm{n}$ el número de no-objetivos, disminuye a medida que aumenta la exposición. El sujeto puede aceptar esta elevada tasa de errores (disminución de la exactitud) o intentar trabajar más despacio, aumentando así la exactitud con la que examina cada signo (disminución de la velocidad).

Lo que se necesita es una técnica que manipule la división de la atención sin cambiar el espaciamiento de los signos - el número de probabilidades de que se den "falsas alarmas». Esta técnica, introducida por Eriksen y Spencer (1969), la han usado Shiffrin y sus colaboradores en una larga serie de experimentos. Un estudio de Shiffrin y Gardner (1972) nos proporciona un buen ejemplo. En cada ensayo se presentaban cuatro letras, por medio de un taquistoscopio, que formaban un cuadrado. Tres eran no-objetivos (e.g. la forma 0): la tarea consistía en localizar e identificar la cuarta, el objetivo (las letras $T \circ F)$. La medida dependiente era la exactitud. En la condición de simultaneidad (SIM) se presentaban los cuatro signos a la vez. En la condición de sucesión (SUC) el ensayo se dividía en dos partes: en la primera exposición se presentaban dos signos (que formaban una diagonal del cuadrado), y en la segunda se presentaban los otros dos. El sujeto sabía de antemano qué diagonal aparecería en cada exposición $\mathrm{v}$ se dejaban 500 milisegundos entre ambas. Al igual que en la condición SIM, el objetivo debía identificarse después de que apareciesen los cuatro signos. Aunque en ambas condiciones se presentaban cuatro signos (y por tanto tenían la misma probabilidad de dar una falsa alarma), la condición SUC exigía una división menor de la atención, ya que en cada momento se examinaban sólo dos signos. El hecho de que en ambas condiciones se obtuviese el mismo 
rendimiento va en contra de la idea de que haya limitaciones en la división de la atención, y en otros experimentos se han obtenido resultados similares utilizando estímulos muy distintos: visuales (Shiffrin, Gardner y Allmeyer, 1973), auditivos (Shiffrin, Pisoni y Castaneda-Méndez, 1974), táctiles (Shiffrin, Craig y Cohen, 1973) y de modalidad mixta (Shiffrin y Grantham, 1974).

Estos resultados son semejantes a los que, a base de variar el tamaño de la presentación, sugieren que la búsqueda se realiza en paralelo. Aparentemente, si la distinción entre objetivos y no-objetivos es simple o es muy conocida, entonces la división de la atención tiene poco o ningún efecto cuando un sujeto inspecciona varios estímulos en busca de un único objetivo.

Es importante que estos resultados sean consistentes con independencia de que la tarea requiera discriminación de formas. El objetivo podría ser un dígito entre un conjunto de letras (Egeth et al., 1972) o un simple toque en la piel (Shiffrin, Craig y Cohen, 1973).

\section{Detección de objetivos independientes}

En otro tipo de experimentos de detección de objetivos, deben identificarse separada e independientemente estímulos simultáneos, teniendo que dar respuestas separadas para cada uno. En la mayoría de estos experimentos se han usado estímulos auditivos. En un.estudio de Ostry, Moray y Marks (1976) se presentaban dos palabras simultáneamente, una a cada oído. Si la palabra presentada al oído izquierdo era un nombre de animal (objetivo) había que presionar una palanca con la mano izquierda. Si la palabra del oído derecho era un nombre de animal, habia que presionar otra palanca con la mano derecha. Podía darse cualquier combinación (ningún objetivo, un objetivo en uno de los oídos $u$ objetivos en ambos oídos) y por tanto podía darse cualquier combinación en las respuestas. Otros muchos experimentos han usado este mismo diseño pero con tonos puros y distinciones muy simples entre objetivos y no-objetivos (e.g., una pequeña diferencia de frecuencia) (Moray, Fitter, Ostry, Favreau y Nagy, 1976; Pohlmann y Sorkin, 1976; Shiffrin y Grantham, 1974; Sorkin, Pohlmann y Gilliom, 1973).

En todos estos experimentos el dividir. la atención produjo disminuciones de rendimiento. La forma exacta de los resultados es especialmente llamativa. Supongamos que los estímulos simultáneos son A y B, pudiendo ser ambos un objetivo o un no-objetivo, y que cada uno tiene su respuesta separada. El rendimiento en un estímulo (A) puede evaluarse en función del evento simultáneo (B). Hay cuatro eventos posibles: aciertos (objetivos correctamente identificados), falsas alarmas (noobjetivos detectados incorrectamente como objetivos), rechazos correctos (noobjetivos correctamente identificados) y omisiones (objetivos detectados incorrectamente como no-objetivos). Este análisis nos revela la existencia de un patrón de resultados que se repite con mucha consistencia. El rendimiento en un estímulo (A) es mejor cuando el estímulo simultáneo es un rechazo correcto, es peor cuando es un acierto o una falsa alarma, $y$ es intermedio cuando es una omisión (Eijkman y Vendrik, 1965; Moray, 1975; Moray et al., 1976; Ostry et al., 1976; Pohlmann y Sorkin, 1976; Sorkin et al., 1973). Con una cantidad adecuada de práctica, el rendimiento en $A$ cuando se dan rechazos correctos simultáneos en $\mathrm{B}$ es tan bueno como si no se estuviese dividiendo la atención (Ostry et al., 1976; Pohlmann y Sorkin, 1976). Pero en cambio esto no se produce cuando el evento simultáneo es un acierto, una falsa alarma o una omisión.

De nuevo, los resultados son los mismos con independencia de que la tarea exija discriminación de formas. Los resultados son similares tanto si los objetivos y no-objetivos se distinguen por una pequeña diferencia de frecuencia (Moray et al., 1976) como si las palabras difieren sólo en su clase semántica (Ostry et al., 1976).

\section{Identificación independiente}

Hasta aquí nuestra discusión sugiere que aunque las disminuciones de rendimiento que se producen con la división de la atención pueden ser pequeñas o nulas cuando los sujetos buscan un único objetivo, puede demostrarse claramente que se da cierto patrón de disminuciones si hay que identificar independientemente como objetivos 0 no-objetivos, estímulos que se presentan simultáneamente y que requieren respuestas separadas. Incluso fuera del contexto de la detección de objetivos parece ser que la regla general sigue siendo que si hay que identificar independientemente estímulos simultáneos con respuestas separadas para cada 
uno, entonces hay que esperar que la división de la atención tenga cierto efecto, a menudo bastante importante.

En apoyo de estos podrían citarse muchos experimentos. En algunos, los estímulos son simples, de forma que esencialmente el sujeto hace juicios psicofísicos simultáneos. Los sujetos de Long (1975), por ejemplo, juzgaban simultáneamente la frecuencia de un tono y la intensidad de una luz, con respuestas escritas separadas para cada una. Los de Lindsay, Taylor y Forbes (1968) juzgaban simultáneamente la intensidad de un tono y la posición de un punto, de nuevo con respuestas separadas para cada una. Otras tareas requieren discriminación simultánea de formas. Los sujetos de Sperling $(1963,1967)$ tenían que identificar todas las letras de una presentación taquistoscópica, informando separadamente de cada una. Los de Broadbent (1958) y Treisman y Geffen (1967) realizaban respuestas separadas ante mensajes presentados de forma dicótica.

Por supuesto que estos experimentos se han realizado con una amplia variedad de estímulos, respuestas, medidas dependientes, etc. Además, uno recibe la impresión de que si hay que identificar independientemente estímulos simultáneos con respuestas separadas para cada uno, entonces casi siempre hay que esperar que el dividir la atención tenga algún efecto (e.g., Allport, 1971; Egeth y Pachella, 1969; Forbes, Taylor y Lindsay, 1967; Massaro y Kahn, 1973; Massaro y Warner, 1977; como excepción puede verse Ériksen y Lappin, 1967). Esto quizá concuerda con las conclusiones obtenidas a partir de los estudios de búsqueda de objetivos, y los sitúa en un contexto más general.

\section{Resumen}

Resumiendo: si los sujetos buscan un único estímulo objetivo (y la distinción entre objetivos y no-objetivos es sencilla o está bien aprendida), puede haber muy poco o ningún decremento en el rendimiento a causa de la división de la atención. Si, por el contrario, hay que identificar independientemente estímulos simultáneos como objetivo o no-objetivo con respuestas separadas para cada uno, entonces la división de la atención produce un patrón específico de efectos. La exactitud con cualquier estímulo dado es peor cuando el otro es un objetivo (o se le confunde con un objetivo). En general, el dividir la atención suele tener efectos importantes cuando hay que identificar estímulos simultáneos con respuestas separadas para cada uno. Estas conclusiones se aplican con independencia de cuál sea la discriminación perceptual que requiera la tarea, desde el juicio psicofísico de una sola dimensión hasta el análisis completo de la forma.

Por supuesto, en esta revisión he considerado experimentos de tipos: muy diferentes, con estímulos, respuestas y medidas dependientes muy distintas. A continuación intentaré confirmar las conclusiones referentes a la búsqueda de objetivos en un contexto experimental muy sencillo. La tarea es visual y requiere cierto grado de discriminación de formas: búsqueda de un dígito objetivo entre un grupo de letras no-objetivo.

\section{DEMOSTRACIONES EMPIRICAS}

Sugiero una regla muy sencilla para las tareas de búsqueda de objetivos en las que los objetivos y no-objetivos o pertenecen a categorías muy distintas y bien conocidas o se distinguen por alguna característica física muy simple. El dividir la atención tendrá efectos sustanciales especialmente cuando pueden darse objetivos simultáneos $\mathrm{y}$ hay que identificarlos independientemente. En cualquier tarea que permita que la ejecución se base en la detección de un único objetivo, el dividir la atención tendrá poco o ningún efecto.

\section{Experimento 1}

Los experimentos $1 \mathrm{a}, \mathrm{b}$ y $\mathrm{c}$ fueron similares. Los sujetos inspeccionaban conjuntos de cuatro signos y buscaban dígitos objetivo entre letras no-objetivo. Para manipular el grado de división de la atención se usó la técnica descrita anteriormente al hablar del trabajo de Shiffrin y Gardner (1972). En la condición SIM se presentaban juntos los cuatro signos, mientras que en la SUC aparecían dos cada vez, imponiendo menos demandas de división de la atención. Las condiciones SIM y SUC se compararon en dos tareas diferentes de búsqueda. En una tarea, los sujetos esencialmente buscaban un único objetivo. En esta tarea se esperaba que la condición SUC tuviese poca o ninguna ventaja. En la otra, había que detectar objetivos independientemente en las dos mitades del conjunto estimular presentado. Se esperaba que la condición SUC 
tuviese una ventaja sustancial, especialmente para cada mitad de los estímulos presentados cuando en el mismo ensayo se detectaba un objetivo en la otra mitad.

\section{Método}

Estimulos. Para el experimento se utilizó un computador PDP-15. Los grupos de estímulos consistian en cuatro signos preparados en un tubo de rayos catódicos (TRC) en forma de cruz (signo más), y el centro de la cruz correspondía al punto de fijación. Cada signo se construyó iluminando los puntos apropiados de una matriz de $7 \times 5$ a una distancia de visión de aproximadamente $36 \mathrm{~cm}$., subtendiendo aproximadamente $.8^{\circ} \times$ $\times 6^{\circ}$ del ángulo visual, centrado a $1.75^{\circ}$ de la fijación. Los signos adyacentes estaban separados de centro a centro por aproximadamente $2.5^{\circ}$. Los signos situados a las 3 y a las 9 formaban el brazo horizontal de la cruz; los de las 12 y las 6 formaban el brazo vertical. La posición de cada signo podía indicarse por medio de una varilla marcadora de $33^{\circ}$ de larga apuntando hacia el signo y centrado a $.83^{\circ}$ de su extremo exterior. Una marca de fijación central también subtendía $.8^{\circ} \times .6^{\circ}$. Una lámpara oscurecida proporcionaba una iluminación de fondo borrosa en una habitación ya de por sí oscura.

Los conjuntos de estímulos consistian en dígitos objetivo entre letras no-objetivo. Los dígitos objetivo aparecían indistintamente en los brazos horizontal y vertical de la cruz. Así, un ensayo podía contener objetivos en ambos brazos, aunque nunca había más de un objetivo por brazo. En cada brazo la probabilidad de aparición de un objetivo era de un tercio. Cuando un brazo contenía un objetivo, su posición en el brazo se determinaba al azar, teniendo cada posición la misma probabilidad de aparición. Las letras se escogían de entre el conjunto $A, E, G, J, M, P$, $\mathrm{T}, \mathrm{X}, \mathrm{y}$ los dígitos de 2-9. Se tomaron independientemente para cada ensayo, con la única condición de que los cuatro signos elegidos fuesen diferentes.

Presentación. Cada ensayo de la condición SIM comenzaba con la marca de fijación central, presentada durante 500 mseg., acompañada durante los primeros 250 mseg. por las cuatro varillas marcadoras. Después de los
500 mseg. se presentaba el conjunto de cuatro caracteres en exposiciones que duraban $\mathrm{T}$ mseg., siendo $\mathrm{T}=\mathbf{2 0}$ en el experimento 1a, y $T=15$ en los experimentos $1 \mathrm{~b}$ y 1c. Este conjunto se reemplazaba inmediatamente por un conjunto correspondiente de cuatro máscaras (manchas de luz rectangulares del tamaño de los signos y que cubrian las posiciones que acababan de ocupar los signos). Estas máscaras se dejaban durante 2000 mseg. al acabar el ensayo e inmediatamente comenzaba el siguiente.

Cada ensayo de la condición SUC se dividía en dos mitades. La primera comenzaba como antes, con una presentación de 500 mseg. de la marca de fijación central. Durante los primeros 250 mseg., esta marca se acompañaba de dos varillas marcadoras que indicaban o el brazo vertical o el horizontal (que se designaba como primer brazo). A los $500 \mathrm{mseg}$. se presentaban los dos signos de este brazo, mientras que en las dos posiciones del otro brazo (segundo) se colocaban las máscaras. Al igual que antes, los signos se reemplazaban por máscaras después de los $\mathrm{T}$ mseg., y el conjunto resultante de cuatro máscaras se dejaba durante $500 \mathrm{mseg}$. En este momento comenzaba la segunda mitad del ensayo. La marca de fijación aparecía durante otros $500 \mathrm{mseg}$., acompañada durante los primeros $250 \mathrm{mseg}$. por varillas marcadoras que indicaban el segundo brazo del conjunto estimular. Después de $500 \mathrm{mseg}$. se presentaban los dos signos de este brazo, mientras que las dos posiciones del primer brazo las ocupaban las correspondientes máscaras. De nuevo, tras $\mathrm{T}$ mseg. se reemplazaban los signos por máscaras, y el conjunto de cuatro máscaras se mantenía durante $2000 \mathrm{mseg}$. Entonces acababa el ensayo e inmediatamente comenzaba el siguiente.

En el experimento la el brazo horizontal fue siempre el primero para todos los sujetos. En los experimentos $1 \mathrm{~b}$ y 1c, el brazo horizontal fue siempre el primero para la mitad de los sujetos; para la otra mitad el primero fue siempre el vertical. Por tanto, los sujetos sabían siempre con anterioridad qué brazo examinar en cada mitad de los ensayos SUC, y aunque al igual que en la condición SIM la decisión se basaba siempre en un total de cuatro signos, sólo se examinaban dos en cada momento. 
Tareas. Había dos tareas diferentes de búsqueda. En la tarea combinada había una sola palanca de respuesta que había que apretar si se detectaba algún objetivo. Ya que los objetivos se presentaban en cada brazo independientemente y con probabilidad igual a $1 / 3$, la probabilidad de que apareciese al menos un objetivo era de $1 / 3+1 / 3-1 / 9=5 / 9$. Bastaba con una simple presión en la palanca, incluso cuando se detectaban objetivos en ambos brazos (esto se aplica también a la condición SUC, en la que se exponían ambos brazos por separado). En principio, esto se consideró equivalente a la búsqueda de un único objetivo. Aunque podian darse objetivos simultáneos, sólo era necesario encontrar uno. Se esperaba que la condición SUC tendría poca o ninguna ventaja sobre la condición SIM (el experimento 2 contiene una tarea en la que, de hecho, no se presentaban objetivos de forma simultánea).

En la tarea separada había que examinar independientemente los dos brazos. Habia una palanca (mano izquierda) para los objetivos detectados en el primer brazo, y otra (mano derecha) para el segundo brazo (para un sujeto dado el brazo que realmente era el primero en la condición SIM, tanto para la asignación de palancas como para el análisis de los datos). Por tanto, en un ensayo cualquiera el sujeto podía apretar ninguna, una o las dos palancas. En esta tarea habia que identificar independientemente los objetivos simultáneos. Se esperaba encontrar una diferencia acusada entre las condiciones SIM y SUC; también se esperaba encontrar una dificultad especial en cada brazo cuando se detectaba simultáneamente un objetivo en el otro.

Se les hizo especial hincapié a los sujetos para que no se preocupasen de la velocidad y se concentrasen en la exactitud. En la condición SUC la respuesta a un objetivo detectado en el primer brazo podía realizarse inmediatamente o después de la presentación del segundo brazo. Los sujetos eran totalmente conscientes de las probabilidades de que apareciese un objetivo y se les animó a que respondiesen acerca de la proporción correcta de ensayos.

Diseño. Los sujetos se tomaron del Departamento de Psicologia de la Universidad de Oregón. Cada uno participó en varias sesiones en días diferentes.

En el experimento la había dos grupos de 8 sujetos cada uno, $10 \mathrm{mu}$ jeres y 6 hombres, de edades comprendidas entre los 18 y los 32 años. A un grupo se le dio la tarea combinada $y$ al otro la tarea separada. Cada sujeto participó en dos sesiones.

En el experimento $1 \mathrm{~b}$ había un único grupo de cuatro sujetos, $3 \mathrm{mu}$ jeres y un hombre, de edades comprendidas entre 21 y 33 años. Cada sujeto participó en 10 sesiones. La mitad de los sujetos realizaron la tarea combinada en las cinco primeras sesiones y la tarea separada en las otras cinco; la otra mitad las realizaron en el orden inverso.

En el experimento 1c había un único grupo de cuatro sujetos, 3 mujeres y un hombre, de edades comprendidas entre 19 y 28 años. Cada uno participó sólo en cinco sesiones de la tarea combinada.

Procedimiento. Cada sesión se dedicaba enteramente o a la tarea combinada o a la tarea separada y tenía dos bloques de la condición SIM y dos de la condición SUC, en orden ABAB o BABA (contrabalanceado entre los sujetos y para cada sujeto alternándolos de una sesión a otra). Cada bloque tenía 5 sub-bloques, el primero de 24 ensayos (de calentamiento) y los otros de 48 ensayos. Cuando aparecía una señal de aviso (se presentaba "simultáneo»o "sucesivo" en el TRC), el sujeto apretaba una palaca para comenzar el subbloque. El primer ensayo comenzaba 500 mseg. después, y los ensayos se continuaban sin pausa tal y como hemos descrito anteriormente. Un tercio de los ensayos de cada subbloque tenía objetivos, independientemente para los brazos horizontal y vertical; además, las secuencias eran aleatorias. Los sub-bloques estaban separados por una corta pausa.

Análisis. Los datos se almacenaron en una cinta magnética y se analizaron por medio de un programa de computador. Para todos los sujetos se consideró la primera sesión (en el experimento $1 \mathrm{~b}$, la primera sesión de cada tarea) como de práctica, y por tanto se descartó. En el resto de las sesiones se descartaron también los 24 ensayos de calentamiento (a partir del comienzo de cada bloque SIM o SUC), y los análisis se basaron, por tanto, en los restantes sub-bloques de 48 ensayos. 
La tarea combinada se evaluó en función del conjunto total de estímulos, tanto en la condición SIM como en la SUC. Por tanto, se anotaba un acierto cuando se presionaba la palanca y había presente (al menos) un objetivo en cualquiera de los dos brazos. Se anotaba una falsa alarma cuando se presionaba la palanca y ningún brazo contenía un objetivo. La tarea separada se puntuó separadamente para cada brazo. Por tanto, se anotaba un acierto en el primer brazo cuando éste contenía un objetivo y el sujeto presionaba la palanca de la mano izquierda, y se anotaba una falsa alarma cuando este brazo no contenía un objetivo y el sujeto presionaba la palanca; ídem para el segundo brazo y la palanca de la mano derecha.

Para cada sujeto y tarea se transformaron las tasas de aciertos y falsas alarmas a las medidas de la teoría de detección de señales, $d^{\prime}$ y $\beta$. Daremos los resultados de estas medidas, pero los experimentos de este artículo se puntuaron también en términos simplemente de porcentajes correctos (media de las probabilidades de los aciertos y los rechazos correctos). En ningún caso se cambió el patrón de resultados (aunque en unos pocos casos se perdió significación estadística). También son necesarias otras precauciones, ya que ni las medidas basadas en la teoría de detección de señales ni las de porcentajes correctos pueden ser ideales. Sin embargo, un resultado que apa- rezca en ambas medidas probablemente será real.

No pueden obtenerse d' y $\beta$ cuando las tasas de aciertos y falsas alarmas son 1.0 ó $.0, \mathrm{y}$ a veces se necesitó agrupar los datos para evitar esto. En concreto, los datos del primero y segundo brazo en la tarea separada a veces hubo que agruparlos de forma que los valores de d' y $\beta$ que se dan, reflejan un promedio aproximado del rendimiento en los dos. Estos casos se mencionan en el texto, y se examinaron siempre los datos para asegurar, en lo posible, que los resultados agrupados reflejasen claramente el rendimiento en cada brazo.

\section{Resultados}

Tarea combinada: objetivos únicos. Para el análisis principal del rendimiento en la tarea combinada se apartaron los ensayos que tenían dos objetivos (uno en cada brazo). Se obtuvo por tanto un porcentaje de aciertos a partir de aquellos ensayos en los que se presentaba un único objetivo y un porcentaje de falsas alarmas a partir de aquellos en los que no se presentaba ninguno. Los valores medios derivados de $d^{\prime}$ y $\beta$ se presentan en la Tabla 1, por separado para los experimentos 1a, b y c. Para los experimentos $1 \mathrm{~b}$ y $1 \mathrm{c}$ se presentan agrupadas las sesiones $2+3$ de la tarea combinada, separadamente de las sesiones $4+5$ agrupadas.

TABLA 1

Experimento 1: valores medios de $d$ y $\beta$ en la detección de un único objetivo en la tarea combinada

\begin{tabular}{ccccc}
\hline \multirow{2}{*}{$\begin{array}{c}\text { Experi- } \\
\text { mento }\end{array}$} & Sesión & Medida & \multicolumn{2}{c}{ Condición } \\
\cline { 4 - 5 } & & & SIM & SUC \\
\hline \multirow{2}{*}{$1 \mathrm{a}^{\mathrm{a}}$} & 2 & $\mathrm{~d}^{\prime}$ & 2.02 & 2.08 \\
& & $\beta$ & 1.10 & .89 \\
$1 \mathrm{~b}^{\mathrm{b}}$ & $2+3$ & $\mathrm{~d}^{\prime}$ & 1.92 & 2.27 \\
& & $\beta$ & 2.55 & 1.74 \\
& $4+5$ & $\mathrm{~d}^{\prime}$ & 2.24 & 2.49 \\
$1 \mathrm{c}^{\mathrm{b}}$ & $2+3$ & $\mathrm{~d}^{\prime}$ & 3.16 & 1.65 \\
& & $\beta$ & 1.59 & 1.87 \\
& $4+5$ & $\mathrm{~d}^{\prime}$ & 1.51 & 2.14 \\
& & $\beta$ & 1.83 & 1.44 \\
\hline
\end{tabular}

Nota. SIM=presentación simultánea; SUC=presentación sucesiva.

- Cada valor es la media de ocho sujetos.

- Cada valor es la media de cuatro sujetos. 
En el experimento 1a los efectos de la división de la atención, que se reflejarían en que los valores de d' serían superiores para SUC que para SIM, son mínimos. La ventaja de la condición SUC se dio sólo en cuatro de los ocho sujetos. A los valores de d' se les aplicó un análisis de varianza (intrasujetos) no siendo significativo el factor condición (SIM versus SUC), $F(1,7)=.3$. Se encontró una tendencia de $\beta$ a ser superior en SIM que en SUC, pero tampoco fue significativa, $F(1,7)=3.7$. Prestaremos poca atención a las diferencias en $\beta$, aunque tendieron a seguir esta dirección.

En el experimento $1 b$ la ventaje de d en la condición SUC fue de .30 . Se aplicó un análisis de varianza (intrasujetos) a los valores de d' con los factores condición (SIM versus SUC) y sesión $(2+3$ versus $4+5$ ). Ningún efecto fue significativo; para el factor condición, F (1, $3)=3.3$. Sin embargo, para cada sujeto en cada nivel de práctica, SUC fue ligeramente superior a SIM. Igualmente, ninguna de las diferencias en $\beta$ fue significativa.

En el experimento 1c la ventaja en d' de la condición SUC fue nuevamente de .30. En un análisis de varianza similar, el efecto de la condición fue significativo, $F(1,3)=19.5, p<.025$. Ningún otro efecto fue significativo, ni tampoco en el análisis correspondiente de $\beta$.

Parece ser que en esta tarea el dividir la atención tiene un ligero efecto, un efecto en d' de algo menos de .3. Aunque es pequeño y (presumiblemente por esta razón) tiene poca fiabilidad, es casi seguro que es real. Más adelante aportaremos más datos en apoyo de esto.

Tarea combinada: objetivos dobles.
Los objetivos dobles ofrecen dos posibilidades potencialmente independientes de realizar una detección correcta. Haciendo ciertas suposiciones, el incremento esperado en la probabilidad de detección es fácil de calcular. Llamemos $p, q$ y $r$ a las probabilidades de respuesta cuando hay 0,1 y 2 objetivos en el conjunto de estímulos. Ahora consideremos un único brazo, el horizontal o el vertical. Puede contener un objetivo y un no-objetivo. Supongamos que en este caso la probabilidad teórica de que el sujeto vea al menos un objetivo en este brazo es $x$. En el caso alternativo, el brazo contiene dos no-objetivos. Llamemos en este caso $y$ a la probabilidad de ver al menos un objetivo en este brazo. Supongamos que los dos brazos se examinan separadamente, independientemente, y con igual exactitud, de forma que el sujeto simplemente responde "objetivo" cuando se percibe un objetivo en cualquiera de ellos. Esto da,

$$
\begin{aligned}
& p=2 y-y^{2} \\
& q=x+y-x y \\
& r=2 x-x^{2}
\end{aligned}
$$

En cada sujeto, los valores de $p$ y $q$ se usaron para derivar $y, x$ y una predicción de $r: r_{\mathrm{p}}$. Los valores medios de $p, q, r$ y $r_{\mathrm{p}}$ se presentan en la Tabla 2. No hubo diferencias grandes o consistentes entre $r$ y $r_{\mathrm{p}}$. Hay que tener cuidado con esto, ya que la estimación $r_{\mathrm{p}}$ se basa en suposiciones (e.g., la misma exactitud en ambos brazos) que no son totalmente correctas. Además, no hay razones claras para dudar de que los objetivos dobles actúen independientemente cuando la tarea es sencillamente detectar al menos un objetivo.

TABLA 2

\begin{tabular}{|c|c|c|c|c|c|c|c|c|c|}
\hline \multirow{3}{*}{$\begin{array}{l}\text { Expe- } \\
\text { rimento }\end{array}$} & \multirow{3}{*}{ Sesión } & \multicolumn{8}{|c|}{ Condición } \\
\hline & & \multicolumn{4}{|c|}{ SIM } & \multicolumn{4}{|c|}{ SUC } \\
\hline & & $p$ & $q$ & $r$ & $r_{p}$ & $p$ & $q$ & $r$ & $r_{p}$ \\
\hline \multirow{3}{*}{$\begin{array}{l}1 \mathrm{a}^{\mathrm{a}} \\
1 \mathrm{~b}^{\mathrm{b}}\end{array}$} & 2 & .180 & .832 & .929 & .957 & .215 & .873 & .953 & .974 \\
\hline & $2+3$ & .140 & .749 & .927 & 919 & .108 & 815 & .938 & .955 \\
\hline & $4+5$ & .088 & .775 & .943 & .941 & .079 & .824 & .986 & .963 \\
\hline \multirow[t]{2}{*}{$1 c^{b}$} & $2+3$ & .162 & .658 & .761 & .792 & .165 & .717 & .797 & .827 \\
\hline & $4+5$ & .117 & .628 & .728 & .715 & .129 & .722 & .781 & .811 \\
\hline
\end{tabular}

Experimento 1: probabilidades predichas y abservadas de una respuesta de detección en la tarea combinada

NorA. SIM=presentación simultánea; SUC=presentación sucesiva.

- Cada valor es la media de ocho sujetos.

- Cada valor es la media de cuatro sujetos. 
Tarea separada: rendimiento general. En la mitad superior de la Tabla 3 pueden verse los valores medios de $d^{\prime}$ y $\beta$ de la tarea separada en el experimento la, basados en los datos agrupados de los dos brazos.

\section{TABLA 3}

Experimento 1a: rendimiento de un único brazo

\begin{tabular}{lllll}
\hline & \multirow{2}{*}{ Tarea } & Medida & \multicolumn{2}{c}{ Condición } \\
\cline { 5 - 5 } & & SIM & SUC \\
\hline Separada & $\mathrm{d}^{\prime}$ & 1.62 & 2.32 \\
Combinada (estimada) & $\beta$ & 1.22 & .99 \\
& $\mathrm{~d}^{\prime}$ & 2.34 & 2.40 \\
& $\beta$ & 1.86 & 1.51 \\
\hline
\end{tabular}

Nota. Cada valor es la media de ocho sujetos. $S I M=$ presentación simultánea; SUC=presentación sucesiva.

Esta tarea sufrió un efecto bastante grande de la división de la atención. d' sufrió una reducción, de SUC a SIM, de .70 . Se realizó un análisis de varian$\mathrm{za}$ sobre los valores de $\mathrm{d}^{\prime}$, siendo la condición (SIM versus SUC) el único factor (intrasujetos). Este fue altamente significativo, $F(1,7)=16.4, p .005$. La tendencia de $\beta$ a ser mayor en SIM que en SUC fue también significativa, $F(1$, 7) $=6.4, \mathrm{p}<.05$.

La mitad superior de la Tabla 4 muestra los datos correspondientes del experimento $1 b$. Se presentan separados los resultados del primero $y$ del segundo brazo. El decremento medio en 'd' de la condición SUC con respecto a la condición SIM fue de .69 , casi exactamente la misma con cada nivel de práctica. Se realizó un análisis de varianza con los valores de d' (entre-sujetos), siendo los factores la condición (SIM versus SUC) y la sesión $(2+3$ versus $4+5)$. Los únicos efectos significativos fueron los de la condición, $\mathrm{F}(1 ; 3)=35.2, \mathrm{p}<.01$, y los de la sesión, $\mathrm{F}(1,3)=22.5, \mathrm{p}<.025$. Ambas condiciones tuvieron mejorías modestas con la práctica. En el correspondiente análisis de varianza de $\beta$ no se encontraron efectos significativos.

Por tanto, el efecto general que tiene el dividir la atención en esta tarea es relativamente llamativo: un efecto en $\mathrm{d}^{\prime}$ de .7 aproximadamente.

Tarea separada: análisis del evento contingente. La exactitud en cada brazo se midió contingentemente al evento concurrente (en el mismo ensayo) del otro brazo (cf. Moray, et al., 1976; Ostry, et al., 1976). Por tanto, la exactitud

TABLA 4

Experimento 1b: rendimiento en un único brazo

\begin{tabular}{|c|c|c|c|c|c|}
\hline \multirow{3}{*}{ Tarea } & \multirow{3}{*}{ Medida } & \multicolumn{4}{|c|}{ Condiciones } \\
\hline & & \multicolumn{2}{|c|}{ Sesiones $2+3$} & \multicolumn{2}{|c|}{ Sesiones $4+5$} \\
\hline & & SIM & SUC & SIM & SUC \\
\hline \multicolumn{6}{|l|}{ Separada: } \\
\hline $\begin{array}{c}\text { Primer brazo } \\
\text { Segundo brazo } \\
\text { Combinada (estimada) }\end{array}$ & $\begin{array}{l}\mathrm{d}^{\prime} \\
\beta \\
\mathrm{d}^{\prime} \\
\beta \\
\mathrm{d}^{\prime} \\
\beta\end{array}$ & $\begin{array}{l}2.00 \\
2.42 \\
2.29 \\
1.81 \\
2.22 \\
4.69\end{array}$ & $\begin{array}{r}2.67 \\
2.90 \\
2.97 \\
.87 \\
2.58 \\
3.08\end{array}$ & $\begin{array}{l}2.26 \\
1.60 \\
2.61 \\
1.04 \\
2.53 \\
5.67\end{array}$ & $\begin{array}{l}2.79 \\
1.70 \\
3.47 \\
1.14 \\
2.79 \\
4.78\end{array}$ \\
\hline
\end{tabular}

Nota. Cada valor es la media de cuatro sujetos. SIM=presentación simultánea; SUC=presentación sucesiva. 
en el primer brazo se midió de forma separada (respectivamente) para los ensayos en los que se produjeron aciertos y rechazos correctos en el segundo brazo, y viceversa. No se obtuvieron las puntuaciones que se dieron con omisiones o falsas concurrentes, pues éstos se produjeron en raras ocasiones.

La Tabla 5 presenta los valores medios de d' y $\beta$ en el experimento 1a, basados de nuevo en datos agrupados del primer y segundo brazos (para realizar este análisis hubo que senarar los datos de un sujeto, ya que incluso después del agrupamiento su tasa de aciertos era de 1.0 en una de las casillas).
Los resultados fueron muy similares a los que anteriormente han encontrado otros autores (e.g., Moray, et al., 1976; Ostry, et al., 1976; Sorkin, et al., 1973). En la condición SIM, el rendimiento fue mucho peor cuando el evento concurrente era un acierto que cuando era un rechazo correcto. Obviamente, esta interacción no se dio en la condición SUC. Los valores de d' se sometieron a un análisis de varianza (intra-sujetos), siendo los factores la condición (SIM versus SUC) y el evento concurrente (aciertos versus rechazo correcto). Los únicos efectos significativos fueron los de la condición, $F(1,6)=14.6, p<.01$. Reali-

TABLA 5

Experimento 1a: análisis del evento contingente en la tarea separada

\begin{tabular}{lccrc}
\hline & & \multicolumn{2}{c}{ Condición } \\
\cline { 3 - 5 } & Evento concurrente & Medida & SIM & SUC \\
\hline Acierto & & & 1.23 & 2.14 \\
Rechazo correcto & d' & 2.58 & 1.74 \\
& d' & 1,82 & 2.08 \\
& d' & 1.08 & .88 \\
\hline
\end{tabular}

Nota. Cada valor es la media de siete sujetos. $\mathrm{SIM}=$ presentación simultánea; $\mathrm{SUC}=$ presentación sucesiva.

zando una comparación planificada, el rendimiento en la condición SIM fue significativamente peor con aciertos concurrentes que con rechazos correctos concuirrentes, $F(1,6)=20.0, p<.005 ; y$ con aciertos concurrentes, el desembeño fue significativamente peor en SIM que en SUC, $F(1,6)=28.4, p<.005$. Con un rechazo correcto concurrente, el desempeño no fue significativamente diferente en SIM y SUC, $F(1,6)=2.2$, pero SUC presentó una pequeña ventaja (una diferencia de 26 en d ). En un análisis similar de $\beta$ no se encontraron efectos significativos.

En la Tabla 6 se presentan los datos correspondientes al experimento $1 \mathrm{~b}$. También en este caso se han agrupado los datos de los dos brazos. El patrón de resultados en d' se repitió y se mantuvo igual en cada nivel de práctica. Los valores de d' se examinaron por me dio de un análisis de varianza (intrasujetos), siendo los factores la condición (SIM versus SUC), la sesión $(2+3$ versus $4+5$ ), y el evento concurrente (aciertos versus rechazos correctos). Los únicos efectos significativos fueron la condición, $F(1,3)=17.6, p<.025$; el evento concurrente, $F(1,3)=50.5, p<.01 ; y$ su interacción, $F(1,3)=15.3, p<.05$. En una comparación planificada, el desempeño en SIM fue significativamente peor cuando el evento concurrente era un acierto que cuando era un rechazo correcto, $F(1,3)=28.6, p<.025$. SUC fue significativamente mejor que SIM con aciertos concurrentes, $F(1,3)=18.7, p<.025 ;$ y casi también con rechazos correctos concurrentes, $F(1,3)=9.3$, $p<.1$. Esta última diferencia se mantuvo, a pesar de ser pequeña, en los cuatro sujetos en las sesiones $2+3$ y en tres de ellos en las sesiones $4+5$. Este pequeño declive debido a la práctica no fue significativo, $F(1,3)=3.5$. En el análisis correspondiente de $\beta$ no se obtuvieron efectos significativos.

Tomados juntos los dos experimentos, la diferencia en d' entre las condiciones SUC y SIM es de aproximadamente 1.0 con aciertos concurrentes y 3 con rechazos correctos concurrentes. Este último efecto, similar al observado en la tarea combinada, aunque sea pequeño es probablemente real. Posteriormente aportaremos más datos en favor de ella.

Comparación entre las dos tareas. Tal y como se han establecido, las medidas del desempeño en las tareas combinada 
TABLA 6

Experimento 1b: análisis del evento contingente en la tarea separada

\begin{tabular}{|c|c|c|c|c|c|}
\hline \multirow{3}{*}{ Evento concurrente } & \multirow{3}{*}{ Medida } & \multicolumn{4}{|c|}{ Condición } \\
\hline & & \multicolumn{2}{|c|}{ Sesiones $2+3$} & \multicolumn{2}{|c|}{ Sesiones $4+5$} \\
\hline & & SIM & SUC & SIM & SUC \\
\hline $\begin{array}{l}\text { Acierto } \\
\text { Rechazo correcto }\end{array}$ & $\begin{array}{l}\mathbf{d}^{\prime} \\
\boldsymbol{\beta} \\
\mathbf{d}^{\prime} \\
\boldsymbol{\beta}\end{array}$ & $\begin{array}{l}1.62 \\
1.60 \\
2.42 \\
2.51\end{array}$ & $\begin{array}{l}2.68 \\
1.86 \\
2.85 \\
2.07\end{array}$ & $\begin{array}{l}1.94 \\
2.20 \\
2.73 \\
1.91\end{array}$ & $\begin{array}{l}3.00 \\
1.07 \\
2.98 \\
1.59\end{array}$ \\
\hline
\end{tabular}

Nora. Cada valor es la media de cuatro sujetos. $S I M=$ presentación simultánea; $S U C=$ presentación sucesiva.

y separada no son directamente comparables. Una refleja las decisiones acerca del conjunto completo de estímulos, y la otra refleja decisiones separadas para cada brazo. Sin embargo, si aceptamos las suposiciones antes descritas, puede usarse el rendimiento en la tarea combinada para derivar una medida de la exactitud en cada brazo aislado. Los valores $p$ y $q$ observados se usaron, por medio de las ecuaciones 1 y 2 y para cada sujeto, para estimar las tasas $x$ e $y$ de aciertos y falsas alarmas para cada brazo, $y$ por tanto los valores $d^{\prime}$ y $\beta$ de cada brazo por separado. (la estimación de $x$ se derivó de la ecuación 2 más que de la 3 , pues el valor $q$ observado se basaba en muchas más observaciones que $r$. Obviamente, la leección suponía muy poca diferencia).

Los valores medios estimados de la tarea combinada en el experimento la se muestran en la mitad inferior de la Tabla 3. De nuevo; la ventaja de SUC fue minima. Para poder realizar una comparación con el rendimiento general en la tarea separada, se realizó un análisis de varianza de los datos d' de la Tabla 3, con el factor (entre-sujetos) tarea (combinada versus separada) y el factor (intra-sujetos) condición (SIM versus SUC). Los únicos efectos significativos fueron la condición, $F(1,14)=$ $=14.1, \mathrm{p}<.005, \mathrm{y}$ la interacción tarea $\times$ condición, $F(1,14)=9.7, p<.01$.

En la mitad inferior de la Tabla 4 se presentan los datos comparables del experimento 1b. Un análisis de varianza similar de d' tuvo los factores (intrasujetos) tarea (combinada versus separada), condición (SIM versus SUC) y sesión $(2+3$ versus $4+5)$, usando para la tarea separada las medias del primer y segundo brazo. Los únicos efectos significativos fueron la condición, $F(1,3)=$
$=14.1, p<.05 ;$ la sesión, $F(1,3)=38.7$, $\mathbf{p}<.01 ; \quad y$ la interacción tarea $\times$ condición, $F(1,3)=14.3, p<.05$.

De nuevo hay que tener cuidado con estas estimaciones del rendimiento en un único brazo en la tarea combinada, basadas como están en suposiciones que indudablemente no son del todo correc. tas. Sin embargo, parece, en principio, que incluso con medidas del desempeño aproximadamente comparables, los efectos de la división de la atención son claramente mayores en la tarea separada que en la tarea combinada.

\section{Discusion}

Resumen. Los resultados obtenidos son más o menos los que esperábamós. En la tarea combinada la división de la atención tuvo un efecto pequeño pero fiable: un decremento de $d^{\prime}$ entre SUC y SIM de aproximadamente 3. Otro punto es que, en esta tarea, los objetivos dobles (uno en cada brazo) actuaron más o menos independientemente. En la tarea separada el efecto de la división de la atención fue mucho mayor: una disminución de d' entre SUC y SIM de aproximadamente .7. En la condición SIM, el rendimiento en los dos brazos no fue en absoluto independiente, siendo mucho peor en cada brazo cuando en el otro se daba un acierto que cuando se daba un rechazo correcto. El decremento de d' entre SUC y SIM fue de aproximadamente 1.0 con aciertos concurrentes, y de 3 con rechazos correctos concurrentes. Con medidas del desempeño aproximadamente comparables, los efectos de dividir la atención fueron significativamente mayores en la tarea separada que en la combinada. 
Réplicas. He replicado varias veces este patrón general de resultados, con varias tareas de búsqueda y un diseño experimental parecido al del experimento 1b. Entre las tareas estudiadas están las siguientes:

1. Búsqueda del dígito objetivo 5 con las letras no-objetivo $S$ y E. Esta tarea era algo especial, puesto que con el conjunto de caracteres usados podía formarse un 5 combinando elementos de una $S$ v una $E$. Aunque importante en otros contextos (Treisman, 1979), esto no pareció tener efectos especiales en este caso.

2. Búsqueda del dígito objetivo 5 con las letras noobjetivos $S$ y G. Aquí no podian combinarse los componentes de los no-objetivos para formar el objetivo, de forma que los objetivos podían encontrarse buscando un único componente estimular (líneas rectas y ángulo en la parte superior). Esto no alteró los resultados.

3. Búsqueda del dígito objetivo 5 , siendo $\mathrm{S}$ todas las letras no-objetivo. Al igual que en los experimentos de Shiffrin (1975), todos los objetivos eran idénticos. De nuevo, los resultados fueron los mismos.

4. Igual que en 2, pero cambiando 'os detalles de presentación en la condición SUC. En cada mitad del ensayo, mientras se presentaban los signos del brazo al que se estaba atendiendo, el brazo no atendido no tenía máscaras sino siu. nos irrelevantes (no-objetivos), sabiendo el sujeto que tenía que ignorarlos. Este control de posibles efectos del enmascaramiento periférico (Estes, 1972) deja los resultados prácticamente inalterados.

En cada uno de estos casos, y en otros pequeños estudios piloto, los resultados fueron muy semejantes a los de los experimentos $1 \mathrm{a}, \mathrm{b}$ y $\mathrm{c}$.

Comparación con la literatura. Obviamente, estos resultados encajan con los revisados al principio del artículo. Sólo un punto exige una mención especial. En mis experimentos he observado consistentemente pequeñas ventajas en la condición SUC, tanto en la tarea combinada como en la separada con rechazos correctos concurrentes. Estas son siempre pequeñas y no significativas en cada experimento, pero en ningún expe rimento se han dado resultados inversos. Indudablemente, ambos efectos son reales.

Otros autores han informado a veces de no haber encontrado absolutamente ningún efecto de la división de la atención cuando los sujetos buscan un único objetivo (e.g., Shiffrin, 1975). Similarmente, cuando hay que examinar los estímulos independientemente, uno $\mathrm{de}$ ellos puede no manifestar ningún efecto de la división de la atención cuando el evento concurrente es un rechazo correcto (Ostry, et al., 1976; Pohlmann y Sorkin, 1976). Aunque estos resultados contrastan con los míos, el contraste no es especialmente sorprendente. Es esperable que no se obtengan a veces estos pequeños efectos de la división de la atención en experimentos concretos. Puede que los oculte el ruido, o posiblemente estén compensados por otros pequeños efectos específicos de los contextos experimentales concretos. Por ejemplo, en la mayor parte del trabajo de Shiffrin sobre la búsqueda de objetivos (e.g., Shiffrin y Gardner, 1972; Shiffrin, Gardner y Allmeyer, 1973) se ha usado una tarea un poco especial. En todos los ensayos hay un objetivo: la tarea consiste en informar de su posición y/o identidad (suelen haber dos objetivos alternativos, e.g., T y F). Consideremos la tarea que les hemos presentado aquí a los sujetos. Supongamos, por ejemplo, que ningun estímulo se parece especialmente a un objetivo. En este caso se elegiría el más parecido al objetivo. Supongamos, por otra parte, que hay dos estímulos que se parecen a un objetivo. También hay que informar de uno sólo. La demanda en este caso no es la de examinar independientemente los diversos estímulos. Es más bien la de compararlos y elegir el más parecido al objetivo. Esta posibilidad está probablemente presente en la mayor parte (si no en todo) del trabajo de Shiffrin. Si la comparación es importante, puede ser que la condición SUC tenga una obvia desventaja. Tiene que ser una comparación a través de los intervalos de observación. Quizá esta desventaja de la condición SUC pueda compensar las ventajas de SUC del tipo que hemos descrito aquí, produciendo a veces incluso un desempeño ligeramente inferior a SIM (Shiffrin y Gardner, 1972; Shiffrin, Gardner y Allmeyer, 1973).

En cualquier caso, el punto importante queda claro. Las ventajas SUC que típicamente hemos descrito aquí, tanto en la tarea combinada como en la separada con un rechazo correcto concurrente, aunque son reales, son sustancialmente más pequeñas que las observadas en general en la tarea separada, y en especial con aciertos concurrentes. Este resultado concuerda perfectamente con el resto de la literatura experimental. 
Práctica. Siempre queda la posibilidad de que los resultados descritos aqui sean específicos de un nivel concreto de práctica, y que con una práctica mayor sean distintos. En los experimentos 1b y lc la práctica tuvo efectos claros, en el sentido de que incluso al final de estos experimentos el desempeño toda. vía iba mejorando. Por el contrario, no se encontró nunca una interacción entre la práctica y otras variables experimentales. Si el patrón de resultados (en oposición al nivel absoluto de rendimiento) estaba cambiando, entonces el cambio era tan pequeño que no se pudo detectar.

En el experimento $1 b$, cada sujeto participó en 10 sesiones, con un total de 8.640 ensayos. Después de este tiem. po, el patrón de resultados seguía siendo similar al observado al principio. Los dos sujetos que realizaban la tarea combinada seguían manifestando una pequeña ventaja en la condición SUC sobre SIM (en las sesiones $9+10$, la diferencia media en d' era de .19). Los dos que participaban en la tarea separada seguían teniendo una ventaja mucho mayor en SUC (diferencia media $=.76$ ), especialmente con aciertos concurrentes (diferencia media $=1.30$ ) y menor con rechazos correctos (diferencia media = $=33$ ). Típicamente, los mayores cambios que se daban con la práctica, si es que se daban, eran Ios del principio. Ya que en el presente caso no-pudieron ser detectados en las 10 primeras sesiones, deben de haber sido muy pequeños (si es que han existido).

A modo de comparación, diré que en los experimentos de Schneider y Shiffrin (1977), los sujetos buscaban dígitos objetivos entre letras (o letras objetivos entre dígitos) durante aproximadamente 7 sesiones en el experimento 1,2 sesiones en el experimento 2, y 10 sesiones en el experimento 3, siendo los sujetos los mismos en todos ellos. La.cantidad total de práctica era como mucho el doble de la que se dio a los sujetos en nuestro experimento $1 \mathrm{~b}, \mathrm{y}$ los autores consideraban a la tarea como claramen. te bien practicada, incluso en su primer experimento.

\section{Experimento 2}

El experimento 1 apoya la regla que hemos sugerido para estas tareas de búsqueda de objetivos. Los efectos de dividir la atención sólo son considera- bles si hay que detectar independientemente objetivos simultáneos. Estos efectos son comparativamente pequeños si la ejecución puede basarse siempre en la detección de un único objetivo.

Aún nos queda una duda. Ciertamente que las tareas combinada y separada tienen una sensibilidad diferente a la división de la atención. ¿Pero esto es así porque la primera permite que la ejecución se base en la detección de un único objetivo? Hay otras diferencias entre estas tareas. La tarea separada requiere simplemente que se transmita más información acerca de los estímulos. La información espacial es particularmente importante. El sujeto debe saber, no sólo si se ha presentado un objetivo, sino que también debe saber dónde se ha presentado. A lo largo de estas líneas, pueden desarrollarse varios argumentos diferentes para explicar los resultados.

Podría ser útil aquí una tarea de búsqueda diferente (1). El sujeto decide si un conjunto de cuatro signos contiene un objetivo o dos. Al igual que en la tarea combinada, sólo es necesario transmitir un bit de información, y ninguna información espacial. Además, ya que la ejecución no puede basarse en la detección de un único objetivo, los efectos de la división de la atención de. ben ser sustanciales.

\section{Método}

Sujetos. En la versión final del experimento participaron seis mujeres y dos hombres, reclutados igual que antes, y de edades comprendidas entre 21 y 30 años. Tres de los sujetos originales tuvieron que ser reemplazados. Dos de ellos dieron tasas de aciertos o de falsas alarmas de 1.0 ó .0 en alguna de las condiciones ex. perimentales. El tercero no entendía las instrucciones.

Tareas. En cada ensayo se presentaban cuatro signos, con exposición SIM o SUC, igual que antes. Nuevamente, los objetivos fueron dígitos y los no-objetivos letras, escogidos entre el mismo conjunto de antes. El sujeto tenia simplemente que determinar el número de objetivos que había en cada ensayo. Había dos tareas. En la mitad o versus is de los ensayos no habia ningún objetivo, y los otros tenían uno. Para indicar el número de objetivos el su-

(1) Estoy muy agradecido a una charla con Angra Tumsuan, la cual me sugirió directamente este experimento. 
jeto presionaba una de las dos palancas de que disponía. El desempeño no podía basarse en la detección de un único objetivo. Para la mitad de los sujetos la palanca izquierda indicaba el número menor de objetivos en cada tarea, y para la otra mitad fue al contrario.

Los objetivos podían aparecer con igual probabilidad -en cada posición, la cual se determinó independientemente y al azar para cada ensayo. En caso de que hubiese dos objetivos, éstos podían aparecer con igual probabilidad en cualquiera de las dos posiciones. Nótese que tanto en la condición SIM como en la SUC los objetivos dobles podían estar en el mismo brazo o uno en cada brazo. Por tanto, el sujeto no tuvo en ningún momento una razón obvia para fijarse especialmente en la posición de un objetivo detectado. Estos no daban ninguna pista acerca del lugar en el que podría aparecer otro objetivo potencial.

Con respecto a lo demás, los estímulos fueron iguales a los de antes. También todos los signos de cada conjunto eran diferentes.

Procedimiento. Cada sujeto participó en tres sesiones. Cada sesión tenía cuatro bloques de ensayos, uno con cada tipo de exposición (SIM o SUC) para cada tarea. La mitad de los sujetos tenía primero los dos bloques de 0 versus $1, y$ después los dos bloques 1 versus 2 , y la otra mitad al contrario. El orden de los tipos SIM y SUC de exposición fueron ABBA o BAAB, contrabalanceando entre los sujetos. El orden de los bloques para cada sujeto era el mismo en las tres sesiones.

Cada bloque tenía cinco sub-bloques, exactamente igual que antes. Se avisaba a los sujetos de que empezaba cada bloque por medio de unas instrucciones que aparecían en el TRC y describían la condición concreta. Las secuencias de ensayos fueron iguales a las de antes, excepto que en cada ensayo la presentación final de las cuatro máscaras se mantenía hasta la respuesta. Entonces un intervalo de 1 seg. en blanco precedía al comienzo del ensayo siguiente. Dentro de cada bloque, exactamente la mitad de los ensayos contenía cada número posible de objetivos, y las secuencias de ensayos eran aleatorias. El tiempo de exposición (T) era de $15 \mathrm{mseg}$.
Análisis. La primera sesión se descartó, pues se la consideró de práctica. Los datos procedentes de los sub-bloques de 48 ensayos de la segunda y tercera sesiones se agruparon. En la tarea 0 versus 1, se consideraba que se había producido un acierto cuando se realizaba la respuesta \&1» y había un objetivo en la pantalla; una falsa alarma era una respuesta a 1s cuando no había objetivos en el ensayo. En la tarea 1 versus 2, un acierto era una respuesta $<2$ realizada en un ensayo que tenía dos objetivos; una falsa alarma era una respuesta $\propto 2$ " realizada en un ensayo que sólo contenía un objetivo. Las tasas de aciertos y falsas alarmas se transformaron a valores d' y $\beta$.

Predicciones. En conjunto, las expectativas eran de que la tarea 0 versus 1 sería comparativamente fácil, y produciría poca diferencia entre SIM y SUC. En esta tarea nunca era necesario detectar independientemente objetivos simultáneos. En realidad no podían darse objetivos simultáneos. La tarea 1 versus 2 se esperaba oue fuese en general más difícil. Incluso en la condición SUC, un tercio (la probabilidad fortuita) de los objetivos dobles tuvo lugar de forma simultánea (en el mismo brazo) y. por tanto, debían de ser difíciles de detectar. Pero se esperaba un rendimiento mucho peor en SIM, en la cual los objetivos dobles exan siempre simultáneos.

En la condición SUC de la tarea 1 versus 2 habría sido interesante comparar la detección de objetivos dobles en el mismo brazo (simultáneos) y en brazos diferentes. Desafortunadamente, esto era imposible, ya que no teniamos disponibles las tasas de falsas alarmas separadas de los dos casos (no se les preguntó a los sujetos, adrede, dónde habían aparecido los objetivos dobles).

Para poner estas expectativas en contexto, el apéndice de este artículo considera las predicciones que haría un modelo en el que no se atribuyasen dificultades especiales a la detección de objetivos simultáneos. Estas suposiciones son que el conjunto de estímulos se examina con igual exactitud en las tareas 0 versus 1 y 1 versus 2, pero un poco mejor en la condición SUC que en la SIM. Desde este punto de vista no podrían predecirse los resultados del experimento 2 . 


\section{Resultados}

En la Tabla 7 se presentan los valores medios de $d^{\prime}$ y $\beta$. Los resultados fueron exactamente los previstos. La tarea 0 versus 1 resultó más fácil que la tarea 1 versus 2 incluso en la condición SUC, y fue mucho menos sensible a la división de la atención. Las diferencias en $d^{\prime}$ entre SUC y SIM fueron de 30 en 0 versus 1 (comparables a las tareas combinadas anteriores), y de .67 en la tarea 1 versus 2.
Se realizó un análisis de varianza sobre los valores de d', con los factores (intra-sujetos) tarea ( $\alpha 0$ versus $1 " v e r$ sus «1 versus 2x) y condición (SIM versus SUC). Se encontraron efectos significativos de la tarea, $F(1,7)=25.7, p<$ $<.005$; la condición, $F(1,7)=39.7, \mathrm{p}<$ $<.001$; y de su interacción, $F(1,7)=10.0$, $p<.025$. En una comparación planificada, 0 versus 1 resultó significativamente mejor que 1 versus 2, tanto en SUC, $F(1,7)=7.2, p<.05$, como en SIM, F (1. $7)=64.0, p<.001$. SIM fue significativa-

\section{TABLA 7}

Ejecución en el experimento 2

\begin{tabular}{|c|c|c|c|}
\hline \multirow{2}{*}{ Tarea } & \multirow{2}{*}{ Medida } & \multicolumn{2}{|c|}{ Condición } \\
\hline & & SUC & SIM \\
\hline $\begin{array}{l}0 \text { versus } 1 \\
1 \text { versus } 2\end{array}$ & $\begin{array}{l}\mathbf{d}^{\prime} \\
\boldsymbol{\beta} \\
\mathbf{d}^{\prime} \\
\boldsymbol{\beta}\end{array}$ & $\begin{array}{l}1.88 \\
2.00 \\
1.07 \\
1.19\end{array}$ & $\begin{array}{l}2.18 \\
2.09 \\
1.74 \\
1.44\end{array}$ \\
\hline
\end{tabular}

Nota. Cada valor es la media de ocho sujetos. $\mathrm{SIM}=$ presentación simultánea; $S U C=$ presentación sucesiva.

mente peor que SUC, tanto en 0 versus $1, F(1,7)=10.5$, $p<.025$, como en 1 versus $2, \mathrm{~F}(1,7)=43.7, \mathrm{p}<.001$.

El análisis correspondiente de $\beta$ no reveló efectos significativos.

\section{Discusión}

Estos dos experimentos apoyan la regla que creemos que sigue el rendimiento en estas tareas de búsqueda. Aparecen dificultades especiales cuando hay que detectar separadamente objetivos simultáneos. Por otra parte, los efectos que tiene la división. de la atención son reales, aunque pequeños. El próximo aspecto a considerar es la importancia que tienen estos resultados con respecto a la distinción entre selección temprana y tardía.

\section{UNA TEORIA DE SELECCION TARDIA}

Una serie de autores recientes que se han sorprendido de la capacidad para encontrar un dígito objetivo entre le tras no-objetivo (o viceversa) independientemente del número de caracteres de la exposición, han hecho una suge- rencia muy simple: a lo mejor es que los objetivos aarrastran» la atención directamente hacia sí (e.g., Hoffman, 1978; Schneider y Shiffrin, 1977; Taylor, 1978). Nunca se aplica atención a los no-objetivos.

Es difícil detectar objetivos simultáneos; sin embargo, el número de noobjetivos simultáneos no tiene ningún efecto. La inferencia obvia es que, en general, los no-objetivos no compiten por ocupar los procesos de capacidad limitada.

\section{La teoria}

\section{LfNEAS GENERALES}

La teoría distingue dos niveles de representación perceptual. El trabajo de identificación y clasificación del estímulo se realiza en el primer nivel, pero los outputs deben pasar a un segundo nivel, a través de un sistema de capacidad limitada, antes de formar una percepción comunicable, o en otras palabras, antes de alcanzar la conciencia.

\section{Promer NIVEL}

En el primer nivel los estímulos simultáneos se examinan en paralelo, y 
el dividir la atención no tiene ningún efecto.

En este nivel la escena perceptual se separa en figura y fondo, y se analizan sus estímulos discretos $u$ objetos. De cada estímulo se analiza totalmente la forma, el color, el tamaño, la posición, etcétera. Ya en este nivel hay mucha información muy conocida que se deduce de la memoria. A un estímulo visual se le designará por su nombre (si el nombre es muy conocido para el sujeto). También se derivan aspectos del significado, como la clasificación en letra o dígito, el significado de una palabra (con cierto nivel de detalle), etc.

La única cosa importante que debemos retener con respecto a este nivel es que aunque se deduzca toda esta información (de forma que las acciones posteriores del sistema pueden depender de ella), nada puede servir todavia como base para la respuesta. Esto se aplica a toda la información: color, posición, forma, significado, etc. Todavía no se ha formado ninguna percepción comunicable de ningún tipo. Ninguna información ha alcanzado la conciencia del sujeto.

La información en el primer nivel es susceptible al enmascaramiento visual, y se va deteriorando; para poder preservarla hay que darle paso.

\section{Programa de selección}

Ya que es un sistema de capacidad limitada el que da paso a los estímulos del primer nivel al segundo, no se puede dar paso a todos los estímulos que componen una única y breve exposición. Por tanto, se necesita algún programa de selección para definir qué estímulos deben elegirse. Potencialmente, cualquier información deducida en el primer nivel podría servir como base para la selección. En la búsqueda de objetivos el propósito normal será elegir sólo los objetivos. Esto será posible si los objetivos son dígitos $-y$ los noobjetivos son letras, pues esta clasificación se realiza en el primer nivel. Por esta razón normalmente pasarán sólo los objetivos, y competirán por entrar en el sistema de capacidad limitada.

\section{El Sistema de capacidad limitada}

Por ahora podemos dejar abierto el tema de la función concreta del sistema de capacidad limitada. Podemos considerarlo simplemente como un proceso que permite que un percepto formado en el primer nivel perceptual pase al segundo. A un estimulo identificado en el primer nivel y elegido según el programa de selección imperante en ese momento, se le introduce en este sistema de capacidad limitada para trasladarlo al segundo nivel.

El sistema de capacidad limitada no puede tratar eficazmente con los estímulos simultáneos, y es la fuente fundamental de todos los efectos de la división de la atención a los que nos referimos aquí. Dos objetivos simultáneos, si hay que detectarlos separadamente, tienen que competir por este sistema. O se les da paso uno detrás del otro o se les da paso juntos con la consiguiente pérdida de exactitud. En cualquiera de los casos, si el tiempo de observación es limitado (y especialmente con enmascaramiento), el rendimiento se deteriorará.

\section{SEGUNDO NIVEL}

La aparición de un estímulo en el segundo nivel crea una percepción comunicable. Por tanto, la llegada de un objetivo a este punto corresponde a su selección, de entre el conjunto estimular, para que entre en la conciencia. En el segundo nivel un estímulo puede almacenarse, repetirse, pueden aplicarse sobre él operaciones aconscientes», etc. Para nuestros intereses actuales, puede comunicarse y se protege del enmascaramiento.

Un estímulo introducido en el segundo nivel es, por supuesto, un percepto basado en el procesamiento del primer nivel. El segundo nivel, por ejemplo, no tiene forma de saber si la información recibida del primer nivel es exacta o no. El único contacto entre el segundo nivel y el mundo externo es por medio del primer nivel.

Probablemente el segundo nivel tenga sus limitaciones de capacidad, en el sentido de que no pueda almacenar, para su comunicación, un número indefinido de estímulos. Por ejemplo, es bien sabido que a medida que aumenta la duración de la exposición, el número de letras comunicadas de una presentación taquistoscópica aumenta rápidamente hasta tres o cuatro, pero después de esto aumenta muy poco a poco. Siguiendo a Sperling $(1963,1967)$, podríamos suponer que el brusco incremento inicial refleja el paso de los items a través de nuestro sistema de capacidad limitada, mientras que el nivelamiento indica que hemos alcanzado la capacidad máxima de un proceso posterior, pro- 
ceso que recibe los items a los que se les deja paso. La suposición primordial de este artículo es que todos estos procesos tardíos, agrupados bajo el nombre de asegundo nivel", pueden ser ignorados para nuestros propósitos actuales. También para alcanzar la capacidad máxima del segundo nivel bastan unos pocos estímulos que haya que comunicar separadamente. Por tanto, cualquier cosa que alcance este nivel puede ser comunicada, y todos los efectos que produce la división de la atención los provoca la competición por ocupar el sistema de capacidad limitada que da acceso a este nivel.

La dificultad está en adquirir información acerca de dos objetivos simultáneamente, y no en la posterior retención de la misma durante el breve período de elección y ejecución de las respuestas.

Esto puede confirmarse por medio de un sencillo experimento. En la tarea separada del experimento 1 , la condición SUC se cambia de tal forma que no se permite dar ninguna respuesta hasta después de que se hayan presentado ambos brazos. Yo he realizado este experimento: el requerimiento de retener la respuesta referente al primer brazo hasta después de que se haya presentado el segundo, y por tanto de retener ambas durante el breve periodo de elección de respuestas, prácticamente no afecta al rendimiento.

\section{Explicación de los datos}

Es importante distinguir dos fuentes de error en los presentes experimentos. Primero, ya que las condiciones de observación son pobres, puede producirse confusión perceptual en el primer nivel. Las letras pueden confundirse con dígitos y viceversa. Sin embargo, ya que el primer nivel es paralelo, la probabilidad de que se produzcan estas confusiones será igual en SIM que en SUC. Con objeto de predecir diferencias entre estas condiciones, podemos ignorar las confusiones al primer nivel perceptual.

La única razón para que SIM sea peor que SUC es que en SIM los estímulos de los dos brazos tienen que competir por el sistema de capacidad limitada. Todo lo que necesitamos considerar para nuestros propósitos actuales es la forma en que, en SIM, esta competición podría distorsionar la información extraída en el primer nivel. Recordemos que, en general, sólo los objetivos normalmente pasarán y competirán por el sistema de capacidad limitada.
Consideremos primero la tarea combinada del experimento 1 , condición SIM. Podría ser que no se detectase ningún objetivo en ningún brazo en el primer nivel. En este caso no pasará nada al segundo nivel, y no se realizará ninguna respuesta de detección. Por el contrario, podría detectarse un único objetivo en el primer nivel, en cualquiera de los brazos. En este caso, este único objetivo será seleccionado y pasará, dando lugar a una respuesta de detección. Podemos suponer que si se detecta más de un objetivo en el primer nivel (e.g., uno en cada brazo), al menos se dará paso a uno. Consideremos ahora la condición SUC. Si en el primer nivel no se detectan objetivos en ningún brazo, entonces no habrá respuesta de detección. Si en el primer nivel se detecta un sólo objetivo en cualquiera de los brazos, entonces habrá una respuesta de detección. Si se detectan más de un objetivo en el primer nivel (e.g., uno en cada brazo), seguirá ocurriendo lo mismo. Según este razonamiento, el rendimiento será igual en SIM que en SUC.

En general, puede verse que, tanto en SIM como en SUC, la probabilidad de que se dé una respuesta de detección es simplemente la probabilidad de que, en el primer nivel, al menos uno de los cuatro estímulos sea identificado como objetivo. Nótese que si la muestra contiene en realidad dos objetivos, uno en cada brazo, esto contribuirá independientemente a la probabilidad de que, en el primer nivel, se identifique al menos un objetivo, y por tanto contribuirá independientemente a la probabilidad de que se produzca una respuesta de detección. Esto fue más o menos lo que pasó en el experimento 1 .

¿Por qué podría en realidad ser peor el desempeño en SIM que en SUC? La posibilidad más obvia es la referente al programa de selección que define que estímulos del primer nivel pueden pasar al sistema de capacidad limitada. Hemos dicho que normalmente sólo los estímulos identificados como objetivos en el primer nivel pasarán y competirán por entrar en el sistema de capacidad limitada. ¿Pero qué pasa si a veces a un estímulo, incluso aunque sea identificado como no-objetivo en el primer nivel, se le deja pasar equivocadamente? Nótese que no estamos hablando de una confusión en el primer nivel. El no-objetivo ha sido identificado adecuadamente como tal, y será percibido como noobjetivo en el segundo nivel. El error está en seleccionar este estímulo (correctamente identificado) para que pase al sistema de capacidad limitada. Si 
esto ocurre de vez en cuando, entonces los objetivos en SIM a veces serán omitidos, dado que los no-objetivos percibidos del otro brazo simultáneamente ocupan el sistema de capacidad limitada. Pero esta pérdida de información que ocasiona la competición por el sistema de capacidad limitada será pequeña, en la medida en que los no-objetivos percibidos puedan excluirse de este sistema, de forma que la diferencia entre SIM y SUC será pequeña, tal y como hemos encontrado.

Consideremos ahora un brazo, A, de la tarea separada. En SIM, un acierto concurrente significa que un objetivo del otro brazo, B, ocupaba el sistema de capacidad limitada. Por lo tanto, el rendimiento en A será pobre, ya que la información referente a $\mathbf{A}$ que alcanzará el segundo nivel será como mucho parcial. Ciertamente el desempeño será mucho peor que en SUC.

Por el contrario, un rechazo correcto concurrente significa que ningún estímulo de B ocupa el sistema de capacidad limitada. De nuevo esto no es definitivo, especialmente porque los no-objetivos de $B$, incluso aunque sean identificados correctamente en el primer nivel, pueden a veces a pesar de ello pasar equivocadamente al sistema de capacidad limitada. Pero en general, el rendimiento en A será relativamente alto, casi tan exacto como en SUC, en la medida en que no se deje pasar a los noobjetivos adecuadamente identificados en el sistema de capacidad limitada.

El análisis de la tarea 0 versus 1 del experimento 2 es similar al de la tarea combinada del experimento 1. La diferencia entre SIM y SUC debería ser pequeña. En la tarea 1 versus 2, habrá una fuerte competición por el sistema de capacidad limitada cuando en la misma exposición se identifiquen dos estímulos como objetivos en el primer nivel. Entonces la posibilidad de que ambos pasen al segundo nivel será más bien baja. En SUC, un tercio de los objetivos dobles se producen en la misma exposición (mismo brazo), mientras que en SIM son simultáneos todos los objetivos dobles. Se esperaba encontrar una diferencia sustancial entre SIM y SUC.

En general, tal y como hemos observado, la división de la atención sólo producirá efectos fuertes cuando haya que detectar separadamente objetivos simultáneos. Sólo en ese caso se producirá una competición seria por el sistema de capacidad limitada.

No sólo en la búsqueda de objetivos, sino en una amplia variedad de situaciones, los efectos de la división de la aten- ción son sustanciales si los estímulos simultáneos hay que identificarlos independientemente, con una respuesta separada para cada uno. Según la presente teoría, estas condiciones implican en realidad que ambos estímulos deben pasar a través del sistema de capacidad limitada. No importa qué discriminación perceptual exija la tarea, desde una discriminación psicofísica unidimensional hasta el análisis completo de la forma. Si los estímulos tienen que identificarse separadamente, ambos tienen que llegar al segundo nivel. Por tanto, la teoría predice adecuadamente tanto los resultados de los presentes experimentos como las principales conclusiones a las que ha llegado la literatura precedente.

\section{ESTRATEGIAS DE SELECCION}

Idealmente, sólo los objetivos necesitan entrar y competir por el sistema de capacidad limitada. Los no-objetivos pueden rechazarse potencialmente después del análisis paralelo del primer nivel. ¿Qué límites hay que poner a esta conclusión? ¿Bajo qué condiciones los no-objetivos no pueden ser rechazados en el primer nivel?

\section{Errores de selección}

En la tarea separada del exoerimento 1 el rendimiento, incluso con rechazos correctos concurrentes, fue muy poco peor en SIM que en SÚC. Esto sugería que incluso aunque los no-objetivos sean identificados correctamente en el primer nivel, a veces entran en el sistema de capacidad limitada, de forma que a veces puede perderse información de un brazo por culpa de la competición por este sistema, incluso aunque en el otro brazo se dé un rechazo correcto. En otras palabras: el programa de selección que define que sólo los objetivos deben pasar del primer nivel al sistema de capacidad limitada no siempre está perfectamente implementado.

En ciertas circunstancias este efecto parece ser bastante grande. Por ejemplo, los sujetos de Ostry, et al. (1976) escuchaban pares de palabras dicóticas e intentaban identificar independientemente objetivos (nombres de animal) en los dos oídos. Después de una práctica abundante, el rendimiento con rechazos correctos concurrentes sufrí poco o ningún efecto de la división de la atención. El desempeño no era mejor cuando ha- 
bía que atender a un sólo oído. Por el contrario, cuando los sujetos tenian poca práctica el efecto del dividir la atención fue sustancial incluso con rechazos correctos concurrentes. Johnston y Heinz (1978) y Treisman y Davies (1973) han encontrado efectos sustanciales semejantes en tareas similares y con poca práctica. Parece, por tanto, que en estas tareas concretas se necesita una gran cantidad de práctica para excluir a los no-objetivos del sistema de capacidad limitada con eficacia. Con poca práctica se consiguen algunos éxitos (la actuación es mejor con un rechazo correcto concurrente que con un acierto concurrente; Ostry, et al., 1976) pero no muchos; incluso la actuación con rechazos correctos concurrentes es sustancialmente peor de lo que sería si hubiera que atender a un sólo oído.

En general, según la presente teoría, el desempeño con rechazos correctos sufrirá los efectos de la división de la atención en la medida en que los no-objetivos percibidos como tales no puedan mantenerse excluidos con eficacia del sistema de capacidad limitada. Probablemente esto variará entre las tareas y los niveles de práctica.

\section{Estrategias alternativas}

En ciertas circunstancias es necesario usar este tipo de argumentos. Todos los intentos de seleccionar sólo los objetivos para que pasen por el sistema de capacidad limitada podrían abandonarse.

Pueden seleccionarse los estímulos de las muestras visuales en base a diferentes características: posición espacial (Posner, Nissen y Ogden, 1978; Sperling, 1960), adyacencia a una varilla marcadora (Averbach y Coriell, 1961; Eriksen y Hoffman, 1973), color, tamaño, etcétera (Von Wright, 1970). Parece probable que después de que los estímulos hayan sido procesados en el primer nivel, puedan implementarse muchos programas de selección diferentes para definir qué estímulos deben pasar al sistema de capacidad limitada. Dejar pasar a los dígitos y rechazar las letras es sólo una estrategia apropiada para una tarea concreta.

La elección de programas de selección puede no ser totalmente libre. Shiffrin y Schneider (1977) nos proporcionan un excelente ejemplo. Los sujetos buscaban dígitos objetivo entre letras no-objetivo o letras objetivo entre dígitos no-objetivo. Después de tener mucha práctica en una de estas tareas, cada sujeto te- nía que realizar la otra. El rendimiento empeoró catastróficamente, y los sujetos decían que no podían ignorar los noobjetivos (previamente objetivos). Aparentemente, la selección de estímulos era en este caso en cierto grado "automátican: esto es, los items con los que se ha practicado mucho siendo objetivos no podian rechazarse a pesar de que las instrucciones habian cambiado.

Sin embargo, dejando aparte estos efectos "automáticos", parece ser que en la búsqueda de objetivos el intentar seleccionar sólo objetivos para dejarlos pasar al sistema de capacidad limitada sería sólo una de las diversas estrategias posibles. Como alternativa, los estímulos de la muestra podrían ser introducidos en el sistema de capacidad limitada siguiendo un orden espacial fijo, tomando los objetivos y no-objetivos de una forma aselectiva. ¿Podría tener ventajas esta alternativa? Evidentemente, sí. Por ejemplo, el intentar seleccionar sólo los objetivos para que pasen por el sistema de capacidad limitada debe de gastar cierto tiempo. Esto significaría que la información entra en el sistema de capacidad limitada más tarde de lo que lo haría de otra forma, y puede pasar menos información antes de que las representaciones del primer nivel se pierdan. Si el criterio de selección de objetivos es muy complejo, entonces podría abandonarse esta selección a causa de su costo. En una situación extrema esto sería imposible. En realidad, tanto los objetivos como los no-objetivos serian introducidos en el sistema de capacidad limitada, seleccionados en función de otra característica (e.g., en un orden espacial fijo).

En estas circunstancias, la descripción de la actuación en la búsqueda de objetivos que hemos hecho en este artículo se rompería. En concreto, no habría razón para que la actuación fuese mejor con rechazos correctos concurrentes que con aciertos, ya que los objetivos y noobjetivos tendrían la misma probabilidad de ocupar el sistema de capacidad limitada. El experimento 3 fue diseñado más o menos como experimento demostrativo para ilustrar este resultado en unas condiciones que complican cualquier intento de seleccionar sólo los objetivos para darles entrada en el sistema de capacidad limitada.

\section{Experimento 3}

En este experimento los sujetos buscaban objetivos que aparecian independientemente en las dos mitades de una 
exposición: izquierda y derecha. Los objetivos simultáneos había que detectarlos separadamente. En una condición se buscaba el mismo objetivo en toda la muestra estimular. En este caso se esperaba el resultado normal: el rendimiento será mejor en cada lado cuando se produzcan rechazos correctos concurrentes que cuando se produzcan aciertos concurrentes. En la otra condición se buscaban objetivos diferentes en los dos lados de la muestra de estímulos. Se esperaba que en esta situación, más compleja, el intentar pasar sólo objetivos al sistema de capacidad limitada sería problemático. En lugar de eso podría darse paso tanto a los objetivos como a los no-objetivos. Podría no haber ninguna o poca diferencia entre la ejecución con rechazos correctos concurrentes y con aciertos concurrentes.

\section{Método}

Sujetos. Se reclutaron dos grupos de 2 sujetos de la misma manera que en el experimento 1. Eran $10 \mathrm{mu}$ jeres y 6 hombres, de edades comprendidas entre 18 y 31 años.

Tarea. Los sujetos buscaban objetivos en un conjunto de cuatro caracteres. Los caracteres eran similares a los utilizados anteriormente, pero ahora dispuestos en un rectángulo cuyo centro coincidia con el punto de fijación. Los dos signos de un lado del punto de fijación estaban separados, de borde a borde, por una distancia vertical de aproximadamente $.6^{\circ} \mathrm{o}$ la anchura de un signo, estando el par centrado a $1.75^{\circ}$ horizontalmente desde la fijación (igual que antes). La marca de fijación central y las condiciones generales de visión eran también iguales a las de los experimentos anteriores.

Había que detectar independientemente los objetivos de los dos lados. El sujeto disponía de una palanca (mano izquierda) para los objetivos de la izquierda, y otra (mano derecha) para los de la derecha.

Los objetivos eran de dos tipos. En un caso el objetivo era el dígito 8 , y los no-objetivos eran los dígitos $3,5,6$ y 9. En el otro caso, el objetivo era la letra G, y los no-objetivos eran las letras $C, O, S$ y $Q$. Cada sujeto tenía dos tareas. Los sujetos del grupo puro buscaba un 8 en ambos lados durante la mitad de la sesión experimental y una $G$ en ambos lados en la otra mitad. Los sujetos del grupo mixto buscaban durante la mitad de la sesión el 8 en la izquierda y la $G$ en la derecha, y durante la otra mitad al contrario. Los no-objetivos de cada lado eran siempre los apropiados para el objetivo que se estaba buscando en ese lado. En todos los casos los objetivos aparecían independientemente en cada lado y con una probabilidad de .5. Nunca aparecía más de un objetivo en un solo lado. Si en un lado aparecía un objetivo, éste se encontraba aleatoriamente en la parte superior o en la inferior, e independientemente para los dos lados cuando ambos contenían objetivos. En cada posición que no contenía un objetivo aparecía un no-objetivo que se elegía al azar de entre los conjuntos posibles, separadamente para cada ensayo, con la limitación (igual que en los experimentos anteriores) de que no hubiesen dos iguales en la misma exposición.

No se manipuló la división de la atención. Los cuatro caracteres se presentaban siempre simultáneamente. Los ensayos comenzaban con una marca de fijación central que duraba $750 \mathrm{mseg}$. Inmediatamente, ésta era reemplazada por los estímulos, y éstos desaparecían (25 mseg. después) de la misma forma que en los experimentos anteriores. Las máscaras permanecían $2 \mathrm{seg}$. en la pantalla y durante este tiempo había que dar la respuesta exacta, sin importar la velocidad. Los objetivos que de hecho tenía la exposición reaparecían entonces durante $500 \mathrm{mseg}$., permitiendo que el sujeto constatase la exactitud de la respuesta. Si el ensayo no contenía objetivos, entonces en este intervalo de feedback se dejaba la pantalla en blanco. Al acabar este intervalo comenzaba inmediatamente el siguiente ensayo.

Procedimiento. Cada sujeto participaba en dos sesiones, cada una de las cuales contenía dos bloques, uno de cada una de las tareas que tenía que realizar el sujeto. Dentro de los grupos puro y mixto se contrabalanceó el orden de las tareas entre los sujetos, pero cada sujeto seguía el mismo orden en cada sesión.

Cada bloque consistía en nueve subbloques; el primero (de calentamiento) tenía 24 ensayos, y los demás 48 cada uno. Una vez presentada la señal aadelante», que aparecía en el TRC (tubo de rayos catódicos), el sujeto apretaba una palanca para comenzar el sub-bloque, el cual empe- 
zaba entonces según el ritmo descrito. Dentro de cada sub-bloque aparecían con igual frecuencia los cuatro eventos posibles (no-objetivo a la izquierda y no-objetivo a la derecha; no-objetivo a la izquierda y objetivo a la derecha; objetivo a la izquierda y no-objetivo a la derecha; objetivo a la izquierda y objetivo a la derecha), pero se aleatorizaron las secuencias.

Análisis. Se analizaron los datos de los sub-bloques de 48 ensayos de ambas sesiones. Se obtuvieron para cada sujeto los valores de d' y $\beta$ para los objetivos 8 y G. Aunque se puntuaron independientemente los dos lados, de nuevo se agruparon los datos para calcular $d^{\prime}$ y $\beta$ (nótese que para el grupo mixto esto significaba el agrupamiento de bloques diferentes de cada sesión).

\section{Resultados}

Los resultados del análisis según los eventos contingentes se presentan en la Tabla 8. Son más o menos los esperados. El grupo puro tuvo mejor rendi-

TABLA 8

Rendimiento en el experimento 3

\begin{tabular}{|c|c|c|c|c|c|}
\hline \multirow{3}{*}{ Evento concurrente } & \multirow{3}{*}{ Medida } & \multicolumn{4}{|c|}{ Objetivo } \\
\hline & & \multicolumn{2}{|c|}{ Sesión 1} & \multicolumn{2}{|c|}{ Sesión 2} \\
\hline & & 8 & G & 8 & $G$ \\
\hline \multicolumn{6}{|l|}{ Grupo puro: } \\
\hline $\begin{array}{l}\text { Acierto } \\
\text { Rechazo correcto }\end{array}$ & $\begin{array}{l}d^{\prime} \\
\beta \\
d^{\prime} \\
\beta\end{array}$ & $\begin{array}{l}1.38 \\
1.54 \\
1.82 \\
1.02\end{array}$ & $\begin{array}{l}1.14 \\
1.68 \\
1.40 \\
1.50\end{array}$ & $\begin{array}{l}1.81 \\
1.50 \\
2.09 \\
1.31\end{array}$ & $\begin{array}{l}1.48 \\
1.96 \\
1.55 \\
1.48\end{array}$ \\
\hline \multicolumn{6}{|l|}{ Grupo mixto: } \\
\hline Rechazo correcto & $\begin{array}{l}\mathbf{d}^{\prime} \\
\beta \\
\mathbf{d}^{\prime} \\
\beta\end{array}$ & $\begin{array}{l}1.16 \\
1.32 \\
1.32 \\
1.13\end{array}$ & $\begin{array}{r}.80 \\
1.43 \\
.70 \\
1.14\end{array}$ & $\begin{array}{l}1.46 \\
1.23 \\
1.56 \\
1.43\end{array}$ & $\begin{array}{r}.99 \\
1.37 \\
.95 \\
1.24\end{array}$ \\
\hline
\end{tabular}

NotA. Cada valor es la media de los ocho sujetos.

miento con rechazos correctos contingentes que con aciertos, mientras que el grupo mixto no. Se realizó un análisis de varianza con los valores de d' con el factor. (entresujetos) grupo (puro versus mixto) y los factores (intrasujetos) evento concurrente (aciertos versus rechazos correctos), objetivo (8 versus G) y sesión (1 versus 2). Lo más importante: se encontraron efectos significativos del evento concurrente, $F(1$, 14) $=11.0, \mathrm{p}<.01$, y de la interacción grupo $\times$ evento concurrente, $F(1,14)=7.3$, $\mathrm{p}<.025$. También produjeron efectos significativos principales el objetivo, $F(1$, $14)=38.3, p<.001, y$ las sesiones, $F(1$, 14) $=13.1, \mathrm{p}<.005$. El rendimiento fue mejor con 8 que con $G$, y mejoró con la práctica. El último efecto significativo fue la interacción evento concurrente $\times$ objetivo, $F(1,14)=7.4, p<.025$. La diferencia entre rechazos concurrentes y aciertos fue mayor con el objetivo 8, aunque no está claro el porqué.

Los sujetos del grupo puro parecieron adoptar rápidamente la estrategia de dejar pasar preferentemente a los objetivos en el sistema de capacidad limitada. La superioridad con rechazos correctos concurrentes sobre los aciertos concurrentes fue ya muy clara desde la primera sesión. En realidad descendió durante la segunda sesión, aunque las interacciones evento concurrente $\times$ sesión, $F(1,14)=2.2$, no fueron significativas. Los datos sugieren, en todo caso, que se dio un declive con la práctica en el paso preferencial de los objetivos. Volveré a esto más adelante.

El análisis correspondiente de $\beta$ no reveló efectos significativos. 


\section{Discusión}

Estos datos indican que habían dos estrategias diferentes en la selección de los datos que se iban a introducir en el sistema de capacidad limitada. En el grupo puro, los objetivos podían elegirse de entre los no-objetivos según un criterio relativamente claro, buscando el mismo objetivo por toda la pantalla. En este caso el rendimiento era mejor con rechazos correctos concurrentes que con aciertos concurrentes, lo cual nos sugiere que se intentaba dejar pasar al sistema de capacidad limitada sólo a los objetivos. En el grupo mixto el criterio para seleccionar los objetivos era más complejo, pues había diferentes objetivos en los dos lados de la exposición. En este caso la actuación era igual con rechazos correctos concurrentes que con aciertos concurrentes, sugiriendo que tanto los objetivos como los no-objetivos accedían al sistema de capacidad limitada.

\section{La literatura sobre búsqueda visual}

Los resultados sugieren un obvio paralelismo con la literatura sobre búsqueda visual. Aquí además parece ser que si el criterio que distingue a los objetivos de los no-objetivos es complejo o no está bien aprendido, entonces puede no ser posible seleccionar sólo objetivos para darles entrada en el sistema de capacidad limitada. Hay que distinguir tres tipos de experimentos sobre búsqueda visual.

\section{Categorización PRActicada}

La primera revisión de literatura se basa principalmente en aquellos casos en los que los objetivos y no-objetivos pertenecen a categorias distintas y bien aprendidas, como, por ejemplo, la búsqueda de dígitos objetivo entre letras no-objetivo. En este caso, la relación entre el tiempo de reacción (TR) y el tamaño de la muestra sugiere alguna aproximación a la búsqueda paralela o independiente. Ya que la distinción entre objetivos y no-objetivos es simple y muy conocida, el intento de seleccionar sólo objetivos para que pasen al sistema de capacidad limitada puede llevarse a cabo con bastante éxito. Esto corresponde a la situación de los presentes experimentos 1 y 2.

\section{Categorización no practicada}

Supongamos que la distinción entre objetivos y no-objetivos no es muy co- nocida. Por ejemplo, podríamos definir como objetivos un conjunto aleatorio de letras, y otro como no-objetivos. En experimentos de este tipo, las relaciones entre el tamaño de la muestra y el TR no nos sugieren la existencia de búsqueda paralela $\mathrm{o}$ independiente. Por el contrario, el TR aumenta a medida que aumenta el tamaño de la muestra (Estes, 1972; Schneider y Shiffrin, 1977).

Parece razonable inferir que en una tarea así es imposible seleccionar sólo objetivos para darles paso al sistema de capacidad limitada. Quizá el tipo de categorización no practicada requerida en esta situación pueda conseguirse sólo por medio de las operaciones aconscientes» que pueden realizarse únicamente cuando un estímulo ha alcanzado el segundo nivel, de forma que tanto los objetivos como los no-objetivos tienen que pasar a este nivel. Por tanto la búsqueda paralela o independiente es imposible. Esta tarea es quizá la que corresponde a las tareas mixtas del experimento 3 .

\section{UN CASO INTERMEDIO}

Hay un caso intermedio entre estos dos. El sujeto sabe de antemano qué objetivo buscar, pero los no-objetivos pertenecen a la misma clase alfanumérica. Por ejemplo, podría buscarse el dígito 8 entre otros dígitos, o la letra $\mathbf{G}$ entre otras letras. Esto corresponde exactamente a las tareas puras del experimento 3 .

Los resultados del grupo puro son interesantes. Durante el primer día hubo una clara ventaja para los ensayos con rechazos correctos concurrentes sobre los que tenían aciertos concurrentes pero, en cualquier caso, ésta pareció declinar (no significativamente) durante el segundo día. Es como si una estrategia inicial dirigida a seleccionar sólo objetivos para darles paso por el sistema de capacidad limitada, fuese después parcialmente abandonada. por ser demasiado costosa o difícil. Por tanto, seleccionar $\alpha$ ochos» de entre otros dígitos o uges» entre otras letras era posible, pero probablemente más difícil que seleccionar dígitos entre letras (estrategia que se mantuvo durante 10 sesiones en el experimento $1 \mathrm{~b}$.

La literatura sobre búsqueda visual sugiere el mismo tipo de resultados intermedios. Cuando se busca un único objetivo posible entre no-objetivos de la misma clase alfanumérica, algunos autores (Corcoran y Jackson, 1977; Egeth, Atkinson, Gilmore y Marcus, 1973) han encontrado rendimientos com- 
parables a los encontrados en la búsqueda de una letra entre dígitos, pero otros no (Ingling, 1972; Jonides y Gleitman, 1972). Esta mezcla de resultados encaja en general con la idea de las tareas del tipo de la realizada por el grupo puro en el experimento 3 que representa un caso intermedio.

\section{Discusión}

El punto importante que surge en esta sección es que la selección preferencial de objetivos es sólo una estrategia para dejar pasar a los estímulos al sistema de capacidad limitada. Probablemente existan otras posibilidades; la más obvia es la selección basada de alguna forma en la forma espacial. Se elegirán estas otras estrategias si el criterio para distinguir los objetivos de los no-objetivos es complejo o está mal aprendido, o si por alguna razón la estrategia de selección preferencial de objetivos es relativamente ineficaz.

Podría haber varias razones para esto. Por ejemplo, en un experimento de Moray et al. (1976), los estímulos eran dos tonos simultáneos, uno de $467 \mathrm{~Hz}$ y el otro de $1510 \mathrm{~Hz}$. Los objetivos eran tonos un poco más sonoros que los noobjetivos. Con una probabilidad general de .01 de que apareciese un objetivo, el rendimiento con cualquiera de los tonos era marcadamente superior con rechazos correctos concurrentes que con aciertos concurrentes, pero este resultado desapareció con una probabilidad de .5 de que apareciese un objetivo. Yo he observado una interacción similar con la probabilidad de los objetivos en un estudio preliminar. de búsqueda de dígitos objetivos entre letras no-objetivos. Aparentemente, a medida que aumenta la probabilidad de los objetivos, el esfuerzo por dejar pasar sólo objetivos al sistema de capacidad limitada a veces pierde fuerza.

Hay una buena razón para que ocurra esto. Las ventajas de la selección preferencial de objetivos (que reduce la competición por el sistema de capacidad limitada) depende directamente de la probabilidad de los objetivos. Dada esta estrategia, el rendimiento en un estímulo se resiente a causa de la competición por el sistema de capacidad limitada sólo si el otro estímulo es un objetivo o se toma como tal por error. Cuanto más probable sea esto, peor será el rendimiento. Esta estrategia sería menos atractiva a medida que aumentase la probabilidad de los objetivos.
La caracterización que he desarrollado en este artículo acerca de la búsqueda de objetivos se aplicará sólo cuando haya un intento de dejar pasar sólo los objetivos al sistema de capacidad limitada. El rendimiento no será mejor con rechazos correctos concurrentes que con aciertos concurrentes $\mathbf{y}$, como en la búsqueda visual no practicada, la división de la atención afectará mucho al rendimiento, incluso aunque éste se base en la detección de un único objetivo.

\section{SELECCION TEMPRANA Y TARDIA}

La teoría que se propone aquí se basa claramente en una idea de selección perceptual tardía. En el primer nivel, o paralelo, del sistema perceptual, antes de alcanzar los procesos de capacidad limitada, hay ya un análisis completo de la forma del estímulo y una extracción de buena parte de su significado. Toda esta información puede servir como base para el acceso selectivo al sistema de capacidad limitada. Los dígitos objetivos pueden ser seleccionados de entre las letras no-objetivos (como en los presentes experimentos), pueden seleccionarse nombres de animales de entre otras palabras (Ostry et al., 1976).

¿Cómo encaja esta teoría con otras teorías de la literatura sobre el tema? En conjunto es consistente con la mayoría de las teorias de la selección tardía (e.g., Allport, 1977; Deutsch y Deutsch, 1963; Hoffman, 1978; Posner, 1978), cada una de las cuales acentúa aspectos distintos, pero incorporando las mismas ideas básicas. Es especialmente parecida a aquellas teorías de la búsqueda de objetivos (e.g., Hoffman, 1978; Shiffrin y Schneider, 1977) que proponen que los objetivos atraen hacia sí la atención o procesamiento de capacidad limitada, y los no-objetivos son rechazados por procesos previos y paralelos. El desacuerdo auténtico es con las teorías de la selección temprana, las cuales suponen que sólo las características físicas simples del estímulo se extraen en el primer nivel o nivel paralelo del sistema perceptual, de forma que sólo estas características pueden servir como base para el acceso selectivo a los mecanismos de capacidad limitada.

Hay varios tipos diferentes de datos que se refieren a la distinción entre selección temprana y tardía. Vamos a verlos a continuación. 


\section{Selección abierta}

Hay una manera muy directa de investigar el acceso selectivo al sistema de capacidad limitada. Se presentan al sujeto varios estímulos simultáneos $\mathbf{y}$ se le pide que seleccione sólo un subconjunto para que lo comunique. El elemento crucial es el criterio en el que hay que basar la selección. Probablemente el rendimiento será mucho mejor si este criterio permite una selección previa al sistema de capacidad limitada, y no hay que hacerla necesariamente después.

Muchos de los primeros experimentos auditivos se basaban en esta idea. Así Treisman (1964 a, 1964 b) presentó a sus sujetos mensajes simultáneos en prosa, de los cuales había que seleccionar uno y repetirlo en seguida (continuamente), es decir, "sombrearlo». La tarea era relativamente fácil si la selección podía basarse en una característica física sencilla del mensaje deseado (e.g., si los mensajes diferían en la voz o llegaban cada uno por un oído). Por el contrario, la tarea era extremadamente difícil cuando no había ninguna de estas distinciones físicas, en cuyo caso había que seguir un mensaje simplemente en base a la continuidad de su contenido.

Obviamente, podía desarrollarse una explicación muy elegante en términos de selección temprana. Antes del sistema de capacidad limitada sólo estaban disponibles las características físicas simples de los mensajes (voz, localización espacial). Si éstas permitían seleccionar el mensaje deseado, entonces sólo había que dar paso a éste por el sistema de capacidad limitada; por el contrario, si la selección dependía del análisis completo de la palabra y de la extracción de su significado, entonces ambos mensajes tenían que pasar por el sistema, y la selección debía realizarse en algún punto posterior.

Sin embargo, posiblemente hubiese podido hacerse igualmente una explicación basada en selección tardía. Ya he considerado anteriormente la posibilidad de que los criterios de selección puedan variar en complejidad. En el experimento 3 , por ejemplo, el grupo puro usó la selección preferencial de objetivos, pero el grupo mixto no. Supongamos que en un experimento de sombreado las palabras de ambos mensajes sean totalmente identificadas (voz, localización, significado, etc...) en el primer nivel perceptual. El mensaje que hay que sombrear deberá ser seleccionado para que pase al sistema de capacidad limitada. Probablemente, la selec- ción será sencilla si siempre se basa en una característica fija del estímulo, como por ejemplo la localización o la voz. Si por el contrario, no se da esta distinción física entre los mensajes, entonces hay que realizar un nuevo juicio de cada palabra del pasaje, para ver si encaia mejor (mejor que la palabra simultánea) en el contexto del material sombreado en este juicio podría ser muy difícil o imposible, de la misma forma que la selección basada en una categorización compleja pareció ser más difícil o imposible en el experimento 3. Con respecto a esto, habrá algunas partes del mensaje en las que no pueda hacerse una selección perfecta, ya que cualquiera de las dos palabras presentadas podría encajar en el contexto del mensaje sombreado. Incluso siguiendo una explicación en términos de selección tardía no podriamos esperar que un problema de selección compleja de este tipo fuese equivalente a la selección basada en alguna propiedad estimular fijar, como la voz o la localización.

Se han realizado experimentos similares con la visión, en la que la tarea a menudo se denomina informe parcial. Los items a informar hay que seleccionarlos de entre una muestra visual en función de la posición espacial (Averbach y Coriell, 1961; Eriksen y Hoffman, 1973; Sperling, 1960), del color (Von Wright, 1970, 1972), de la clase alfanumérica (Sperling, 1960), etc... En algunos de estos experimentos, la señal indicadora de los items que hay que comunicar se da después del final del estímulo, pero probablemente antes del deterioro en la memoria icónica; para nuestros objetivos actuales esto es indiferente.

Supongamos que una muestra tenga ocho caracteres, cuatro hay que comunicarlos (objetivos) y cuatro hay que ignorarlos (no-objetivos), y que en promedio los sujetos informan tres de los cuatro objetivos, probabilidad correcta $=.75$. Este rendimiento del informe parcial se compara con una condición de informe total en la que los sujetos intentan comunicar los ocho signos. Supongamos que en promedio pueden comunicarse cuatro signos en el informe total, probabilidad correcta $=.5 \mathrm{La}$ diferencia entre estas dos probabilidades correctas se llama superioridad del informe parcial.

Al igual que en los experimentos de sombreado, ha sido fácil encontrar resultados consistentes con la selección temprana. Las superioridades demostradas por el informe parcial han sido 
mayores cuando la selección para el informe parcial se basaba en alguna característica física simple del estímulo, como la posición espacial o el color, y menores cuando se basaba en la clase alfanumérica (selección de dígitos y rechazo de letras, o vice versa). De nuevo, la selección basada en un análisis total de la forma del estímulo se muestra relativamente ineficaz.

Por el contrario, no debemos esperar que el rendimiento sea exactamente igual con todas las pistas de selección (y de hecho la posición espacial a menudo es más efectiva que el color). Aunque se ha. sugerido que la selección basada en la clase alfanumérica no da superioridad en el informe parcial, esto ciertamente no es así (Dick, 1971; Sperling, 1960; : Von Wright, 1970, 1972). La diferencia con la selección basada en la posición espacial o en el color parece ser cuantitativa más que cualitativa.

Hay, además, una dificultad general con este tipo de comparaciones. Supongamos que la pista de selección es el color. La selección será menos eficaz a medida que la diferencia de color entre los items seleccionados y rechazados sea menor. Von Wright (1970) ha demostrado exactamente este tipo de cambio continuo en la eficacia de la selección a medida que cambia la discriminabilidad de la clave. Por tanto; cualquier elección de una diferencia de color concretá (o señal basada en la posición es: pacial) para compararla con la selección basada en la clase alfanumérica es arbitraria. Los intentos de comparar la efícacia de la selección con tipos completamente diferentes de señal de selección se enfrentan siempre con esta dificultad lógíca (Johnstọn y Heinz, 1978; Keren, 1976).

En conjunto, por tanto, no es inconsistente sugerir que en todos estos experimentos de informe parciál la selección precede al sistema de capacidad limitada, pero es más eficaz cuando se basa en las señales concretas de localización espacial o color que se han usado en lós experimentos que cuando se basa en la clase alfanumérica.

De hecho, algunos experimentos de informe parcial apoyan directamente el punto de vista de la selección tardía. En un experimento de Duncan (nota 1), se presentaban a los sujetos conjuntos de ocho caracteres en forma circular: cuatro dígitos (para informar) y cuatro letras (que había que ignorarlas), en orden aleatorio. En las exposiciones de duración más corta normalmente se informaba de uno o dos items como mucho, pero incluso en este caso se daba una marcada superioridad en el informe parcial. La probabilidad de informar un dígito concreto correctamente era mayor en el informe parcial que en el total. En estas condiciones de exposición, parece ciertamente que el principal límite del rendimiento estriba en la introducción de items en el sistema de capacidad limitada antes de perderlos ( $y$ no en la memoria posterior o en los procesos del output), de forma que una superioridad en el informe parcial -nos indica que hay una selección efectiva en este nivel (véase también Allport; 1977).

Las teorías de la selección temprana (e.g., Broadbent, 1971; Treisman, 1964 b) dan una respuesta típica a este tipo de hallazgos: la superioridad del efecto parcial puede deberse a efectos producidos por el criterio, Cuando la tarea consiste en informar sólo de los dígitos pueden darse incluso dígitos de los que la evidencia perceptual no es grande (llegando en casos extremos a la adivinación). Obviamente, este efecto : del criterio podría incrementar la probabilidad: de informes de dígitos correctos. Sin embargo esto haria también que aumentasen las falsas alarmas, y en el experimento de Duncan (1) no se encontraron datos que apoyasen esto. Por tanto, la explicación basada en efectos del criterio no parece afortunada.

En general, por consiguiente, ni los experimentos de sombreado ni los de informe parcial aportan datos claros en contra de la selección tardía, si acaso, es más bien al contrario.

\section{Detección de objetivos durante el sombreado}

Treisman y Geffen (1967) y Treisman y Riley (1969) han descrito dos experimentos importantes. En el de Treisman y Geffen los sujetos recibían mensajes en prosa simultáneos y de forma dicó tica. El mensaje de un oído (mensaje primario) había que sombrearlo. Además ciertas palabras objetivo (e.g., la palabra golpe) que aparecían en cual, quiera de los dos oídos había que reconocerlas golpeando la mesa. Ya que los objetivos los emitía siempre la misma voź que decía el resto del mensaje, su detección no podía basarse en característica física alguna, sino que requeria

(1) Ducan, J.: Partial reports based on colour and on alphanumeric class: Evidence for late selection. Manuscript submitted for publication. 
un análisis de la identidad de la palabra. Se les recalcó a los sujetos que el sombreado era la más importante de las tareas. El resultado fue que los objetivos se detectaron mucho menos en el mensaje secundario que en el primario.

La teoría desarrollada en este artículo predice directamente este resultado, siempre y cuando supongamos que el mensaje primario sobrecargó demasiado el sistema de capacidad limitada como para permitir que pasase al mismo una parte grande del mensaje secundario. Ya que la tarea más importante era sombrear el mensaje primario, puede suponerse que aproximadamente todas las palabras de este mensaje pasaron por el sistema de capacidad limitada, y que por tanto llegaron a la conciencia. Así, cualquier objetivo que apareciese en este mensaje debería detectarse. Consideremos, por el contrario, lo que pasó con los objetivos del mensaje secundario. Incluso aunque fuesen identificados como objetivos en el primer nivel podía no pasar al sistema de capacidad limitada, ya que lo más importante era dejar pasar la palabra simultánea del mensaje primario, pudiendo así seguir sombreando. La detección de objetivos en el mensaje secundario resultaría infrecuente, tal y como se constató.

El experimento de Treisman y Riley (1969) fue similar; excepto en que al detectar objetivos el sujeto tenía que parar el sombreado. Algunos objetivos los pronunciaba, al igual que antes, la misma voz que decía el mensaje; y de nuevo éstos se detectaron mucho menos en el mensaje secundario: que en el primario. Otros objetivos los pronunciaba una voz distinta, y en éstos el ren. dimiento fue máximo en ambos mensajes.

Consideremos primero el caso de los objetivos dichos con la misma voz. El encontrar una detección peor en el mensaje secundario que en "el primario es un poco embarazoso para la presente teoría. Mientras no se producían objetivos en el mensaje secundario debían haber entrado las palabras del mensaje primario en el sistema de capacidad limitada, pudiendo así continuar el sombreado (y detectar los objetivos del mensaje primario). Pero cuando se producía un objetivo en el mensaje secundario debía ser identificado como tal en el primer nivel, . y ya que el sombreado debía detenerse (ésta es. la diferencia crucial con el experimento de Treisman y Geffen, 1967), ya no era necesario dejar pasar más palabras del mensaje primario por el sistema de capacidad limitada. En realidad deberia pasar el objetivo secundario, pudiendo realizarse así una respuesta de detec ción. Idealmente, la detección debería de. haber sido igualmente buena en am. bos mensajes.

Sólo podemos recurrir a la siguiente idea, discutida varias veces anteriormente: algunas veces puede ser que incluso aunque sea en el primer nivel, los estímulos sean identificados total y exactamente, a pesar de lo cual el programa de selección que define qué estímulos deben pasar al sistema de capacidad limitada no está bien implementado. En este experimento la selección se realizaba con presiones de tiempo, ya que los mensajes eran continuos. Durante un período largo de tiempo había que tomar las palabras del mensaje primario, y de repente y de forma: inesperada había que tomar un objetivo del mensaje secundario. Aparentemente, esto fallaba a menudo, ya que incluso aunque fuesen identificados en el primer nivel, los objetivos del mensaje secundario no pasaban por el sistema de capacidad limitada ni alcanzaban la conciencia del sujeto. En realidad el no-objetivo simultáneo del mensaje primario era el que pasaba, de forma que el sombreado continuaba normalmente. Se gún la presente teoría, ésta es la única forma de explicar la' pobre detección de objetivos 'secundarios.

¿Entonces, por qué no pasó lo mismo cuando los objetivos diferían en voz del resto del mensaje? Ś́lo podemos suponer que la diferencia de voz usada por Treisman y riley (1969) era tan grande que en este caso la elección de los items que debían entrar en el sistema de capacidad limitada era casi perfecta. En realidad era posible dejar paso a pa: labras del mensaje primario un rato, y entonces desviarse con confianza cuando se producía un cambio de voz en el secundario. He apuntado, en conexión con el trabajo sobre el informe parcial, que el proceso dè selección de items para darles paso por el sistema de capacidad limitada no será igualmente eficaz con cualquier pista de selección. Las pistas que se han usado normalmente en el informe parcial, basadas en la posición espacial o en el color, parecen más efectivas que la clase alfanumérica, incluso aunque la clase alfanumérica se: conozca de hecho (como yo supongo) tras el procesamiento del primer nivel. Igualmente, aquí la diferencia de voz usada por Treisman y Riley parece haber sido una pista más efectiva que la identidad de la palabra, in- 
cluso aunque supongamos que ambos se conocían en el primer nivel.

No es necesario resaltar el carácter embarazosamente ad hoc de esta explicación. Pero hay que añadir otro punto. Underwood y Moray (1971) repitieron el experimento de Treisman y $\mathrm{Ri}$ ley (1969), pero usaron una diferencia de voz probablemente menos llamativa (aunque seguía correspondiendo a una diferencia de sexo), ya que incluso la detección de objetivos que diferían en voz del resto del mensaje no alcanzaba su máximo. Estos objetivos se detectaron con menor frecuencia en el mensaje secundario que en el primario. En este caso, por tanto, parecía ser que incluso los objetivos definidos por la voz no serán en principio detectados de forma diferente que los objetivos definidos por la identidad de la palabra, en línea con la presente teoría.

\section{Disociaciones}

Quizás la evidencia más clara de que se da una identificación detallada de incluso los estímulos que no llegan a pasar por el sistema de capacidad limitada nos la proporcionan ciertos tipos de disociación entre el informe explícito y otros criterios de identificación. En un experimento típico de este tipo el sujeto sombrea un mensaje auditivo presentado con la suficiente rapidez como para asegurar (o al menos eso es lo que se espera) que ninguna otra información pueda pasar por el sistema de capacidad limitada. Como confirmación hay que constatar que después los sujetos no son capaces de informar del contenido de un mensaje simultáneo no atendido. Sin embargo, hay otros criterios que nos indican que sí se identifica.

Los más conocidos son los experimentos con respuestas galvánicas de la piel (RGP) condicionadas. Primero se condiciona a los sujetos (con un shock eléctrico) a dar una RGP a ciertas palabras o clase de palabras objetivo. Posteriormente pueden obtenerse estas RGP incluso aunque los objetivos se produzcan en el mensaje no atendido durante el sombreado (Corteen y Dunn, 1974; Corteen y Wood, 1972; Moray, 1969; Von Wright, Anderson y Stenman, 1975; los resultados contradictorios informados por Wardlaw y Kroll, 1976, han recibido críticas metodológicas de Forster y Govier, 1978). Una fuerte evidencia de que esto depende de un análisis completo de los significados de las palabras del mensaje no atendido nos la proporciona la generalización de las RGP a sinónimos del objetivo (Von Wright, et al., 1975), o el que aparezcan las RGP más frecuentemente cuando la palabra crítica está dentro, y no fuera, del contexto (sintáctico o semántico) del mensaje no atendido (Forster y Govier, 1978).

En la misma línea, puede demostrarse que las palabras no atendidas influyen en la identificación del mensaje aten dido. El sombreado se realiza más despacio cuando la palabra simultánea no atendida es un sinónimo de la palabra que hay que sombrear (Lewis, 1970; Treisman, Squire y. Green, 1974). Una palabra no atendida puede influir en la interpretación de una frase ambigua a la que se ha atendido (Mackay, 1973).

Estos resultados nos recuerdan mucho a los que a veces se han obtenido con exposiciones taquistoscópicas muy breves. En un experimento de Allport (1977), por ejemplo, se presentaban dos palabras taquistoscópicamente. La que se presentaba en el punto de fijación era la que había que comunicar; normalmente los sujetos ni siquiera eran conscientes de la otra. Sin embargo, el rendimiento se veía facilitado cuando las dos palabras tenían una relación asociativa. Como también sugiere la literatura sobre defensa pereceptual (e.g., Erdelyi, 1974), el significado de una palabra parece a veces estar disponible para algunas partes del sistema, aunque ni su identidad ni incluso su existencia puedan ser informadas.

Este tipo de resultados sugieren claramente que las palabras que nunca llegan a la conciencia pueden no obstante ser identificadas totalmente, conclusión que obviamente es consistente con las teorias de la selección tardía. Aparentemente, una palabra totalmente identificada en el primer nivel del sistema perceptual, aunque no llegue a pasar por el sistema de capacidad limitada puede a pesar de ello ser la responsable de una RGP, o puede incluir de varias formas en la identificación de otras palabras. Probablemente estos efectos tengan lugar realmente en el primer nivel, y por tanto fuera de la conciencia.

Hay un problema potencial con el trabajo con sombreado. Debe establecerse mediante algún criterio que la palabra supuestamente «no atendida» realmente no pase por el sistema de capacidad limitada. Aunque normalmente las palabras no pueden ser comunicadas al final del sombreado, siempre queda la posibilidad de que en realidad estén cayendo rápidamente en el 
olvido. Quizás la mejor respuesta a esta crítica la proporcionaron Corteen y Dunn (1974). En un experimento como el de Treisman y Riley (1969), el sujeto tenía que detener el sombreado inmediatamente cuando detectaba una palabra objetivo (la palabra a la que se había condicionado una RGP) en cualquiera de los dos mensajes. Aunque según este criterio los objetivos no atendidos normalmente no eran detectados, a pesar de ello producían RGPs. Este tipo de disociación entre la respuesta explícita y otros criterios de identificación proporcionan un fuerte apoyo a la idea de que se produce una identificación completa de la palabra sin conciencia, exactamente como esperaríamos desde el punto de vista de la selección tardía.

\section{Resumen}

En conjunto, los datos de la literatura son consistentes con la selección tardía. Es siempre importante resaltar que el proceso que selecciona a los estímulos para darles paso por el sistema de capacidad limitada no operará necesariamente con una exactitud perfecta, o con la misma eficacia sea cual sea la pista de selección. A menudo, por el contrario, será relativamente lento e inexacto. Teniendo esto presente, no hay razones claras para suponer que la selección basada en la forma sea de una clase diferente que la selección basada en características simples, como la posición espaciál o el color.

\section{UNA TEORIA ALTERNATIVA}

Una parte considerable de los argumentos de este artículo en favor de la selección tardía se han basado en el hecho de que en la búsqueda de objetivos el rendimiento puede ser mejor con rechazos correctos concurrentes que con aciertos concurrentes. Moray ha ofrecido una explicación muy distinta de este resultado (e.g., Moray et al., 1976).

En la teoria de Moray la información acerca de estímulos simultáneos está disponible durante un intervalo fijo. Durante este intervalo hay que examinar los estímulos uno por uno, pero la cantidad de tiempo para cada uno es variable. Específicamente, la información sobre un estímulo se va acumulando hasta que se identifica este estímulo con un grado (preespecificado de certeza ( $c f$. los familiares modelos de decisión estadística de Laming, 1968; Stone, 1960; etc.) y entonces se examina el otro. Una suposición crucial es que el sistema está predispuesto hacia los objetivos. El requisito de certeza es mucho mayor para una respuesta de objetivo que para una de no-objetivo. En consonancia, hay que acumular una cantidad grande de información acerca de un estímulo, A, antes de que el sistema acepte la decisión de que es un objetivo, usando una buena proporción del intervalo de observación total, y dejando poco tiempo para otro estímulo, B. El rendimiento con $\mathrm{B}$ será menos exacto con un rechazo correcto concurrente que con un acierto.

Un problema que tiene esta explicación es que no aclara los resultados del experimento 3. No está claro por qué el patrón de resultados debe cambiar cuando se buscan diferentes objetivos en diferentes partes de la muestra. Aunque podría darse una explicación ad hoc, la predicción ciertamente no se sigue de forma natural de lo anterior.

El segundo problema es más serio. La suposición fundamental (que el sistema está predispuesto hacia los objetivos) implica que los valores observados de $\beta$ en estos experimentos deberian ser mayores de uno. Aunque en el trabajo de Moray esto suele ser así, ya que los objetivos son más bien improbables, en otras ocasiones los resultados han sido distintos. Sorkin y sus colaboradores han obtenido de forma consistente mejores rendimientos con rechazos correctos concurrentes que con aciertos, incluso cuando la probabilidad del objetivo era de .5 (e.g., Pohlmann y Sorkin, 1976; Sorkin et al., 1973) condiciones en las que no deberíamos esperar que $\beta$ fuese mayor de uno, como de hecho lo confirman los datos de Sorkin (e.g., Pohlmann y Sorkin, 1976; Sorkin, Pohlmann y Woods, 1976), al menos en algunos sujetos. En el presente trabajo, los valores de $\beta$ normalmente han sido mayores de uno (ya que los objetivos solían ser más bien improbables), pero no siempre. Un sujeto del experimento 1b es especialmente llamativo: a lo largo de cinco sesiones experimentales esta persona estuvo consistentemente predispuesta hacia los objetivos, aunque, con igual consistencia su rendimiento en SIM fue mejor con rechazos correc. tos concurrentes que con aciertos (una diferencia media en d' de .68 , en comparación con un valor medio de .69 para los cuatro sujetos). Este tipo de datos son inconsistentes con la explicación de Ostry et al. (1976). El encontrar me- 
jores rendimientos con rechazos correctos concurrentes que con aciertos no depende de la predisposición hacia los objetivos.

\section{ESPECULACIONES}

Como la presente teoría afirma, las operaciones del : primer nivel son enteramente paralelas. La división de la atención no tiene absolutamente ningún efecto hasta después del punto de la identificación total del estímulo. La justificación de esto es que sólo los objetivos parecen hacer grandes demandas a algún sistema de capacidad limitada. Por tanto, los objetivos tienen que ser distinguidos de los no-objetivos en paralelo, en un punto anterior.

Además, podría haber algún límite de capacidad incluso en el primer nivel. Incluso en la tarea combinada del experimento 1, y también en la tarea separada con rechazos correctos concurrentes, había una pequeña ventaja de SUC sobre Sim. Yo atribuí esto a la posibilidad de que incluso no-objetivos identificados exactamente podrían a veces acceder al sistema de capacidad limitada después del primer nivel, pero, es igualmente posible que incluso el primer nivel no sea enteramente paralelo. Incluso en éste podría haber un cierto límite en la capacidad (produciendo una pequeña ventaja de SUC también la tarea combinada, y en la tarea separada con rechazos correctos concurrentes) además de la posterior y más acusada limitación, tras el rechazo de los noobjetivos. Johnston y Heinz (1978) pre sentan una teoria similar a ésta. Lo que los datos presentes sugieren realmente es que el límite principal en la percepción de estímulos simultáneos se produce después de la identificación completa del estímulo.

Una cuestión distinta es el grado en que las operaciones del primer nivel son «automáticas». ¿Actúan siempre de una forma fija, completa e independiente del contexto de la tarea o de las metas o intenciones del sujeto? Posner (1978), en concreto, ha desarrollado una explicación de este tipo. La implicación es, por ejemplo, que siempre que se presente un estímulo, su identidad, nombre, y quizás varios aspectos de su significado, se deducen siempre totalmente, independientemente de su ubicación actual en los intereses del sujeto. La primera posibilidad de realizar un «control estratégico», esto es, de que los objetivos actuales influyan sobre el curso del procesamiento del estímulo, aparecería por tanto en la elección de los items que se van a introducir en el sistema de capacidad limitada. Esta idea tiene una elegancia atractiva, y será in. teresante ver hasta qué punto puede aceptarse.

¿Cuál es la naturaleza del sistema de capacidad limitada? ¿Es imposible en algún sentido para el control estratégico: que he mencionado tener in sistema que asegure que sólo los estímu: los seleccionados, los que en ese mo mento tienen importancia, sean admitidos en el procesamiento consciente posterior para acomodarlos en el plan y el contexto de la tarea presente? (Posner, 1978), o chay alguna función perceptual específica, como la formación de un percepto unificado a partir de las diversas propiedades del estímulo (forma visual, nombre, significado) extraídas en el : primer nivel? (Allpòrt, 1977): ¿El sistema de capacidad limitada es de modalidad específica o común a todas las modalidades?: Ciertamente, los efectos de la división de la atención pueden surrgir con estímulos de diferentes modalidades (e.g., Lindsay et al., 1968; Long, 1975), y en la búsqueda de obje: tivos puede ser que el rendimiento sea peor en la modalidad A cuando simultáneamente en la modalidad $B$ hay un acierto en vez de un rechazo correcto (Eijkman y Vendrik, 1965; Shiffrin y Grantham, 1974). Por el contrario, algunos experimentos sugieren que los efectos de la división de la atención son generalmente menores con modalidades distintas que con una sola modalidad (Treisman y Davies, 1973).

¿A qué se le da paso por el sistema de capacidad limitada?, ¿a una repre sentación completa del estímulo, . que incluye por ejemplo información acerca del color, el tamaño, y otros atributos que podrían ser irrelevantes para la tarea concreta?, ¿cuáles son las aunidades a las que se les da paso? Por ejemplo, si una persona identifica la forma de un objeto, ise da paso también al color por el sistema de capacidad limitada y queda por tanto éste disponible para ser comunicado sin costos adicionales? (Allport, 1971; Lappin, 1967). Muy similar es el activo debate en la literatura sobre la percepción de las palabras: ¿en qué unidades se perciben y protegen del deterioro las palabras $y$ sus letras componentes? (McClelland y Johnston, 1977; Reicher, 1969). 


\section{Apéndice}

Supongamos que el conjunto de estímulos se examina con igual exactitud en las dos tareas del experimento 2, un poco más exactamente en SUC que en SIM. ¿Qué predicciones se siguen de una simple consideración de los diferentes procesos de decisión requeridos en las dos tareas?

Consideremos un único signo de la presentación. Sea $b$ la probabilidad de un acierto con un único signo (este signo percibido como objetivo, siéndolo de hecho) y $f$ la probabilidad de una falsa alarma con un único signo (este signo percibido como objetivo, cuando en realidad es un no-objetivo). Supongamos que los cuatro signos de la presentación se examinan separada $e$ independientemente $y$ con igual exactitud (sin atribuir dificultades especiales a la percepción de objetivos simultáneos).

Supongamos que en la tarea 0 versus 1 el sujeto responderá: «0» si no ve objetivos, y \&1» en caso contrario. Llamemos $P(a, b)$ a la probabilidad de la respuesta $a$ habiendo $b$ objetivos en la presentación. Entonces,

$$
\begin{aligned}
& \mathbf{P}(0,0)=(1-f)^{4} \\
& \mathbf{P}(0,1)=(1-f)^{3} \cdot(1-b)
\end{aligned}
$$

Para la tarea 1 . versus 2 , supongamos que el sujeto responderá $\$ 1 »$ si percibe ninguno o un objetivo, y «2» en caso contrario. Habiendo un objetivo, hay tres posibilidades en la muestra para dar una falsa alarma con signo único, y una para dar un acierto con un único signo. La respuesta \&\$: se realizará si hay cero falsas alarmas de signo único, o una falsa alarma y una omisión de signo único:

$$
P(1 ; 1)=(1-f)^{3}+3 f(1-f)^{2}(1-b)
$$

Dados dos objetivos, hay dos posibilidades de una falsa alarma con un único signo y dos de un acierto. La respuesta $« 1 »$ se realizará si hay cero falsas alarmas de signo único y menos de dos aciertos de signo único, o una falsa alarma y. cero aciertos de signo único:

$$
\mathrm{P}(1,2)=(1-f)^{2}\left(1-b^{2}\right)+2 f(1-f)(1-b)^{2}
$$

A partir de estas ecuaciones se realizó una sencilla simulación: por computador. Los valores de d' y $\beta$ en las dos tareas se obtuvieton a partir de valöres asignados de $b$ y $f$. Para todos los valores razonables de $b$ y $f$.el resultado fue el mismo: Aunque la tarea 1 versus 2 era un poco menos exacta que la tarea 0 versus 1 , la diferencia 'en d' nunca excedió de 3 y. en general fue más pequeña (compárese con las diferencias observacas de .44 y $: 81$ en las condiciones SUC y SIM respectivamente). Para simular el cambio de SUC a SIM se asignaron valores de partida de $b$ y. $f$, y entonces : se introdujeron decrementos en $b$ y/o incrementos en f. Dependiendo de los valores de partida, cualquiera de las tareas podía ser un poco más sensible:a estos cambios, pero la diferencia era siempre pequeña. Dado que el cambio gẹneral de d' en 0 versus 1 tenía que ser pequeño; el cambio en 1 versus 2 fue normalmente el mismo dentro de .1. Aparentemente, los resultados del experimento 2 no pueden explicarse en estos términos.

Este análisis está limitado por la suposición de que los signos son examinados separada e independientemente $\mathrm{y}$ con igual exactitud. Por el contrario, la mayor parte del presente trabajo sugiere que estas suposiciones son bastante exactas para la tarea 0 versus 1 . En concreto, unas suposiciones' similares predijeron bastante bien la probabilidad de un acierto con dos objetivos en la muestra en la tarea combinada del experimento 1.

\section{Referencias}

AlLPORT; D. A.: «Parallel encoding within and between elementary stimulus dimensions», Perception \& Psychophysics, 10, 104-108, 1971.

AlLPORT, D. A:: «On knowing the meaning of words we are unable to report: The effects of visual masking». In S. DornIC (Ed.), Attention and performance VI, Hillsdale, N. J.: Erlbaum, 1977.

Averbach, E., \& CORIEll, A. S.: «Short term memory in vision», Bell Systems Tecbnical Journal; 40, 309-328, 1961. 
Broadbent, D. E.: Perception and communication, London: Pergamon Press, 1958.

Broadbent, D. E.: Decision and stress, London: Academic Press, 1971.

Corcoran, D. W. J., \& JACxson, A.: «Basic processes and strategies in visual search». In S. Dornic (Ed.), Attention and performance VI. Hillsdale, N. J.: Erlbaum, 1977.

CORTEEn, R. S., \& DunN, D.: «Shock-associated words in a nonattended message: A test for momentary awareness», Journal of Experimental Psychology, 102, 1.143-1.144, 1974.

CORTEEN, R. S. \& WoOD, B.: «Autonomic responses to shock-associated words in an unattended channel», Journal of Experimental Psycbology, 94, 308-313, 1972.

Deutsch, J. A., \& Deutsch, D.: «Attention: Some theoretical considerations», Psycbological Review, 70, 80-90, 1963.

Dick, A. O.: «On the problem of selection in short-term visual (iconic) memory. Canadian Journal of Psychology, 25, 250-263, 1971.

EgeTH, H., AtKInson, J., GilmoRe, G., \& Marcus, N.: Factors affecting processing mode in visual search», Perception \& Psychophysics, 13, 394-402, 1973.

Egetr, H., Jonides, J., \& W WLL, S.: «Parallel processing of multielement displays», Cognitive Psychology, 3, 674-698, 1972.

Egeth, H., \& PACHELla, R.: «Multidimensional stimulus identification», Perception \& Psychophysics, 5, 341-346, 1969.

ErJKMan, E., \& Vendrix, A. J. H.: "Can a sensory system be specified by its internal noise?", Journal of the Acoustical Society of America, 37, 1.102-1.109, 1965.

Erdelyi, M. H.: «A new look at the new loon: Perceptual defense and vigilance», Psychological Review, 81, 1-25, 1974.

ERIKSEN, C. W., \& Hoffman, J. E.: «The extent of processing of noise elements during selective encoding from visual displays», Perception \& Psychophysics, 14, 155-160, 1973.

ERIKSEN, C. W., \& LAPPIN, J. S.: «Independence in the perception of simultaneously presen. ted forms at brief durations", Journal of Experimental Psychology, 73, 468-472, 1967.

ERIKSEN, C. W., \& SPENCER, T.: «Rate of information processing in visual perception: Some results and methodoligical considerations», Journal of Experimental Psychology Monograph, 79 (2, Pt. 2), 1969.

Estes, W. K.: "Interaction of signal and background variables in visual processing», Perception \& Psychopbysics, 13, 278-286, 1972.

Forbes, S. M., TAYLOR; M. M., \& LindSaY, P. H.: Cue timing in a multi-dimensional detection task», Perceptual and Motor Skills, 25, 113-120, 1967.

Forster, P. M., \& GoviER, E.: «Discrimination without awareness? Quarterly Journal of Experimental Psychology; 30, 289-295, 1978.

Hoffman, J. E.: «Search throug a sequentially presented visual display», Perception \& Psychopsysics, 23, 1-11, 1978.

INGLING, N. W.: "Categorization: A mechanism for rapid information processing», Journal of Experimental Psycbology, 94, 239-243, 1972.

Johnston, W. A., \& HeInz, S. P.: «Flexibility and capacity demands of attention», Journal of Experimental Psychology: General, 107, 420-435, 1978.

Jonides, J., \& Gleitman, H.: «A conceptual category effect in visual search: $O$ as letter or as digit», Preception \& Psychopbysics, 12, 457-460, 1972.

KeELe, S. W.: Attention and buman performance, Pacific Palisades, Calif.: Goodyear, 1973.

KEREN, G.: «Some considerations of two alleged kinds of selective attention», Journal of Experimental Psychology: General, 105, 349-374, 1976.

LAMING, D: R. J.: Information theory of choice reaction times. London: Academic Press, 1968.

LAPPIN, J. S.: "Attention in the identification of stimuli in complex displays», Journal of Experimental Psychology, 75, 321-328, 1967.

LEWIS, J. L.: «Semantic processing of unattended messages using dichotic listening», Journal of Experimental Psychology, 85, 225-228, 1970.

LINDSAY, P. H., TAYloR, M. M., \& Forbes, S. M.: «Attention and multi-dimensional discrimination», Perception \& Psychophysics, 4, 113-117, 1968.

LoNG, J.: «Reduced efficiency and capacity limitation in multidimencional signal recognition», Quarterly, Journal of Experimental Psycbology, 27, 599-614, 1975.

MACKaY, D.: "Aspects of the theory of comprehension, memory, and attention», Quarterly Journal of Experimental Psychology, 25, 22-40, 1973.

Massaro, D. W., y KaHN, B.. J.: «Effects of central processing on auditory recognition», Journal of Experimental Psycbology, 97, 51-58, 1973.

MASSARO, D. W., \& W WRNER, D. S.: «Dividing attention between auditory and visual perception», Perception \& Psychophysics, 21, 569-574, 1977.

McClelland, J. L., \& Johnston, J. C.: «The role of familiar units in perception of words and nonwordsm, Perception \& Psychophysics, 22, 249-261, 1977.

Moray, N.: Listening and attention Harmondsworth, England: Penguin Books, 1969. 
Moray, N.: «A data base for theories of selective listening». In P. M. A. Rabbitt \& S. Dornic (Eds.), Attention and performance V. London: Academic Press, 1975.

Moray, N., Fitter, M., Ostry, D., Favreau, D., \& Nagy, V.: "Attention to pure tones», Quarterly Journal of Experimental Psychology, 28, 271-283, 1976.

NeISsER, U.: Cognitive psychology. New York: Appleton-Century-Crofts, 1967.

OSTRY, D. MORAY, N., \& MARKS, G.: «Attention, practice, and semantic targets», Journal of Experimental Psychology: Human Perception and Performance, 2, 326-336, 1976.

PohlmanN, L. D., \& Sorkin, R. D.: «Simultaneous three-channel signal detection: Performance and criterion as a function of order of report», Perception \& Psychophysics, 20, 176$179,1976$.

PoSNER, M. I.: Chronometric explorations of mind. Hillsdale, N. J.: Erlbaum, 1978.

POSNER, M. I., NISSEN, M. J., \& OGDEN, W. C.: «Attended and unattended processing modes: The role of set for spatial location». In H. L. Pick \& E. J. Saltzman (Eds.), Modes of perceiving and processing information. Hillsdale, N. J.: Erlbaum, 1978.

REICHER, G. M.: «Perceptual recognition as a function of meaningfulness of stimulus material», Journal of Experimental Psychology, 81, 275-281, 1969.

SchNeIDER, W., \& SHIFFrIN, R. M.: «Controlled and automatic human information processing: I. Detection, search, and attention», Psychological Review, 84, 1-66, 1977.

SHIFFrin, R. M.: «The locus and role of attention in memory systems». In P. M. A. Rabbitt \& S. Dornic (Eds.), Attention and performance V. London: Academic Press, 1975.

Shiffrin, R. M.; Craig, J. C., \& CohEN, U.: «On the degree of attention and capacity limitations in tactile processing", Perception \& Psychophysics, 13, 328-336, 1973.

SHIFFrIN, R. M., GARDNER, G. T.: «Visual processing capacity and attentional control», Journal of Experimental Psychology, 93, 72-83, 1972.

Shiffrin, R. M.; Gardner, G. T., \& Allmeyer, D. H.: «On the degree of attention and capacity limitations in visual processing», Perception \& Psychophysics, 14, 231-236, 1973.

ShIFFrin, R. M., \& Grantham, D. W.: «Can attention be allocated to a sensory modality?», Perception \& Psycbopbysics, 15, 460-474, 1974.

Shiffrin, R. M.; Pisoni, D. M., \& Castaneda-Mendez, K.: «Is attention shared between the ears?, Cognitive Psychology, 6, 190-215, 1974.

SRIFFrIN, R. M., \& SCHNEIDER, W.: «Controlled and automatic human information processing: II. Perceptual learning, automatic attending, and a general theory», Psychological Review, 84, 127-190, 1977.

Sorkin, R. D.; Pohlmann, L. D., \& Gilliom, J.: «Simultaneous two-channel signal detection: III. 630 and $1400 \mathrm{~Hz}$ signals», Journal of the Acoustical Society of America, 53, 1045-1051, 1973.

Sorkan, R. D.; PohlmanN, L. E., \& Woods, D. D.: «Decision interaction between auditory channels», Perception \& Psychopbysics, 19, 290-295, 1976.

SPERLING, G.: «The information available in brief visual presentations», Psychological Monographs, 74 (11, Whole No. 498), 1960.

SPERLING, G.: «A model for visual memory tasks», Human Factors, 5, 19-31, 1963.

SPERLING, G.: «Successive approximations to a model for short-term memory. In A. F. Sanders (Ed.)", Attention and performance I, Amsterdam, North-Holland, 1967.

STONE, M.: «Models for choice reaction time», Psychometrika, 25, 251-260, 1960.

TAYLOR, D. A.: «Identification and categorization of letters and digits», Journal of Experimental Psychology: Human Perception and Performance, 4, 423-439, 1978.

Treisman, A. M.: «Effects of irrelevant material on the efficiency of selective listening», American Journal of Psychology, 77, 533-546, 1964 (a).

Treisman, A. M.: «Verbal cues, language, and meaning in selective attention», American Journal of Psychology, 77, 206-219, 1964 (b).

TreIsman, A. M.: «The psychological reality of levels of processing. In L. S. Cermak \& F. I. M. Craik (Eds)», Levels of processing and buman memory, Hillsdale, N. J.: Erlbaum, 1979.

Treisman, A. M., \& Davies, A.: «Divided attention to ear and eye». In S. Kornblum (Ed.), Attention and performance IV, London, Academic Press, 1973.

Treisman, A. M., \& Geffen, G.: «Selective attention: perception or response?», Quarterly Journal of Experimental Psychology, 19, 1-17, 1967.

Treisman, A. M., \& Riley, R. G.: «Is selective attention selective perception or selective response? A further test», Journal of Experimental sycbology, 79, 27-34, 1969.

Treisman, A. M.; Squire, R., \& Green, J.: «Semantic processing in dichotic listening? A replication», Memory \& Cognition, 2, 641-646, 1974.

Treisman, A. M.; SYKes, M., \& Gelade, G.: «Selective attention and stimulus integration». In S. Dornic (Ed.), Attention and performance VI, Hillsdale, N. J.: Erlbaum, 1977.

UnDERwoon, G., \& Morax, N.: \&Shadowing and monitoring for selective attention», Quarterly Journal of Experimental Psychology, 23, 284295, 1971. 
Von Wright, J. M.: «On selection in visual immediate memory». In A. F. Sanders (Ed.), Attention and performance III, Amsterdam, North-Holland, 1970.

VoN WRIGHT, J. M.: «On the problem of selection in iconic memory»; Scandinavian Journal of Psycbology, 13, 159-171, 1972.

Von Wrigat, J. M.; Anderson, K., \& STEnman, U.: «Generalisation of conditioned GSRs in dichotic listening». In P. M. A. Rabbit \& S. Dornic (Eds.), Attention and performance V, London, Academic Press, 1975.

WARDLAW, K. A., \& KROLI, N. E. A.: «Autonomic responses to shock-associated words in a nonattended message: A failure to replicate», Journal of Experimental Psychology: Human Perception and Performance, 2, 357-360, 1976. 